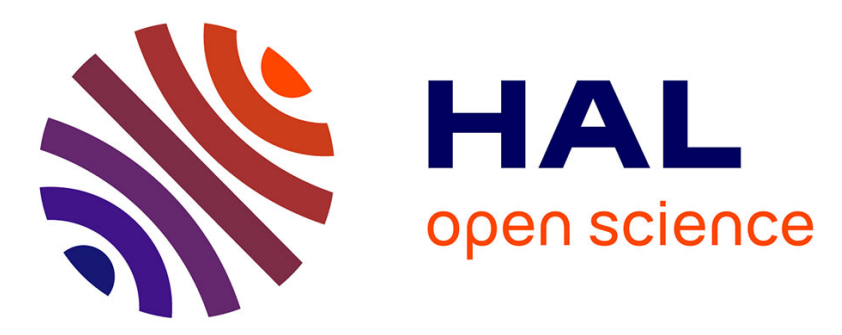

\title{
Return Mapping Algorithms (RMAs) for Two-Yield Surface Thermoviscoplastic Models Using the Consistent Tangent Operator
}

Grégory Antoni, Frédéric Lebon, Thierry Désoyer

\section{- To cite this version:}

Grégory Antoni, Frédéric Lebon, Thierry Désoyer. Return Mapping Algorithms (RMAs) for Two-Yield Surface Thermoviscoplastic Models Using the Consistent Tangent Operator. International Journal of Nonlinear Sciences and Numerical Simulation, 2018, 19 (7-8), pp.681-697. 10.1515/ijnsns-2017-0073 . hal-02021047

\section{HAL Id: hal-02021047 \\ https://hal.science/hal-02021047}

Submitted on 11 Jun 2019

HAL is a multi-disciplinary open access archive for the deposit and dissemination of scientific research documents, whether they are published or not. The documents may come from teaching and research institutions in France or abroad, or from public or private research centers.
L'archive ouverte pluridisciplinaire HAL, est destinée au dépôt et à la diffusion de documents scientifiques de niveau recherche, publiés ou non, émanant des établissements d'enseignement et de recherche français ou étrangers, des laboratoires publics ou privés. 


\section{Return Mapping Algorithms (RMAs) for Two-Yield Surface Thermoviscoplastic Models Using the Consistent Tangent Operator}

\begin{abstract}
The return mapping algorithms (RMAs) presented here are designed for use with pressure-dependent thermoviscoplastic constitutive models involving irreversible effects associated with solid-solid phase transformations. During the volume solid-solid phase transformations occurring under mechanical loads, an "anomalous" plasticity, the so-called "transformation-induced plasticity” (TRIP), is generated at much lower stress levels than those related to the yield stress of the material in the context of the classical plasticity. TRIP mechanisms are superimposed on the classical plasticity which is liable to occur in the case of metallic materials. Based on a non-standard generalized material framework, two different models are presented in which an "associative" plastic flow is introduced in the context of classical plasticity and a "non-associative" flow rule in the context of TRIP-like plasticity. In this paper, a complete algorithmic treatment of these two rate-dependent constitutive models is therefore proposed with the associated consistent tangent operator for dealing the quasi-surface irreversible solid-solid transformations which can appear in metal alloys during specific thermomechanical solicitations. The predictive abilities of the presented numerical procedure for modelling this kind of the irreversible solid-solid transformations involving two plasticity processes are tested and assessed by performing a two-
\end{abstract}

\footnotetext{
*Corresponding author: Gregory Antoni, Laboratoire de Mécanique et d'Acoustique, CNRS, UPR 7051, Aix-Marseille Université, F-13453 Marseille Cedex 13, France, E-mail: antoni.gregory@yahoo.fr Fréderic Lebon, Laboratoire de Mécanique et d'Acoustique, CNRS, UPR 7051, Aix-Marseille Université, F-13453 Marseille Cedex 13, France, E-mail: lebon@lma.cnrs-mrs.fr

Thierry Désoyer, Laboratoire de Mécanique et d'Acoustique, Centrale Marseille, CNRS, UPR 7051, Aix-Marseille Université, F-13453 Marseille Cedex 13, France,

E-mail: desoyer@lma.cnrs-mrs.fr
}

dimensional finite-element analysis on some numerical examples.

Keywords: transformation-induced plasticity, quasisurface irreversible solid-solid phase transformations, return mapping algorithms, consistent tangent operator, finite-element analysis

\section{Introduction}

The return mapping algorithms (RMAs) are widely used in non-linear structural problems (see [1-4]) for providing accurate, efficient and robust iterative methods associated with numerical integration procedure $[5,6]$. RMAs have been classically presented for solving rateindependent (see [7-9]) and rate-dependent (see [10-12]) elastoplastic problems, and their field of application has also been advantageously extended to non-associated plasticity rules (see $[13,14]$ ) and arbitrary yield criteria without losing their unconditional stability. In order to preserve a quadratic convergence rate with the use of Newton-Raphson method, a consistent tangent operator (CTO) (see $[2,15]$ ) - which has also been called the consistent tangent modulus or linearization modulus - is usually employed with RMAs.

In this paper, two thermomechanical models are developed for predicting the behaviour of materials undergoing quasi-surface irreversible (permanent) solidsolid phase transformations which are also known as "Tribological Transformation of Surface" (TTS) (see for example [16]) or "Tribological Surface Transformations" (TSTs) (see [17]). Over the years, some of the French railroad network's rails have been affected by the physical changes of TSTs type on the tread of rails in straight line. Although the physical origin of TSTs has not yet been clearly established, it seems likely that the mechanical loads combined with the thermal effects resulting from the wheel/rail contact may play a crucial role (see [18]). The two thermomechanical models presented here are able to take into account the initiation and development of these TSTs, especially in the immediate vicinity of the 
top of the rails on which the trains run (see [19]), based on the main assumption that the friction occurring in the wheel/rail contact area - where the thermal effects are considerable - is strongly associated with the mechanical loading mainly applied, i.e., that TSTs are due to thermomechanical coupling processes (see [17, 20]). During the solid-solid phase transformations which occur under mechanical loads, an "anomalous" plastic flow, which is also known as TRIP (transformation-induced plasticity) (see [21-25]), generally occurs even at much lower stress levels than the initial yield stress (the softest phase) of the material in question. Indeed, this TRIP-like mechanism can be superimposed on the classical plasticity behaviour which are always present in metallic materials. For several years now, TRIP processes have been widely studied and successfully modelled (see, for example, [26-30]).

In the previous studies, the theoretical point of view of these thermomechanical models has been mainly addressed. Especially, the admissible thermodynamic feature has been investigated (for example, see [20] for a first tentative model and [31, 32] for two other models strongly inspired by the former). Derived from TRIP mechanism, these models have been extended to include thermomechanical coupling so that they can be used to account for TSTs. The present study focuses more specifically on the numerical point of view on these models involving both TRIP-like and classical plasticity processes. The implementation of these models is based on a RMA-type procedure (see for example $[1,3,4]$ ) adapted here to take into account TSTs including these two irreversible processes (TRIP-like and classical plasticity). A CTO (see [4]) is also proposed for using with the RMAs. This paper is organized as follows: (i) the constitutive equations governing these two thermomechanical models are presented in Section 2, (ii) the numerical integration associated with the proposed models is developed in Section 3, (iii) the relevance of this numerical procedure in terms of predictive abilities is tested and discussed by performing a two-dimensional finite-element analysis on some examples in Section 4.

\section{Thermoviscoplastic constitutive models: continuum framework}

\subsection{State equations}

This section deals with the constitutive equations governing two thermomechanical models involving irreversible solid-solid phase transformations and the effects associated with these processes. Within a continuum framework (see $[33,34]$ ) and assuming the presence of small perturbations, two types of state variables are introduced here: (i) the "observable variables" which are: (i)-(1) the absolute temperature $T$; (i)-(2) the total secondorder symmetric strain-tensor $(\boldsymbol{\epsilon})$ which can be written in terms of thermoelastic ( $\boldsymbol{\epsilon}^{e l}$ and $\boldsymbol{\epsilon}^{\text {th }}$ ) and (visco)inelastic $\left(\boldsymbol{\epsilon}^{p c}\right.$ and $\left.\boldsymbol{\epsilon}^{p z}\right)$ strain variables such as $\boldsymbol{\epsilon}=\boldsymbol{\epsilon}^{e l}+\boldsymbol{\epsilon}^{\text {th }}+$ $\boldsymbol{\epsilon}^{p c}+\boldsymbol{\epsilon}^{p z}$; (ii) the "internal variables" for describing the irreversible solid-solid phase transformations and the classical hardening of the material: (ii)-(1) $z=\frac{\rho^{d}}{\rho} \in[0,1]$, which is the mass fraction of the daughter phase (where $\rho^{d}$ and $\rho$ are the partial mass density of the daughter phase and the total mass density, respectively); (ii)-(2) $v$, which is an isotropic hardening variable associated with classical plasticity.

Each of the above strain tensors, $\boldsymbol{\epsilon}$ (resp. $\boldsymbol{\epsilon}^{*}$ with $*=e l, t h, p c, p z)$ is decomposed into its spherical $\frac{1}{3} \operatorname{Tr}(\boldsymbol{\epsilon}) \boldsymbol{G}$ (resp. $\frac{1}{3} \operatorname{Tr}\left(\boldsymbol{\epsilon}^{*} \boldsymbol{G}\right)$ ) and deviatoric parts $\boldsymbol{e}$ (resp. $\boldsymbol{e}^{*}$; see [35]):

$$
\begin{gathered}
\boldsymbol{\epsilon}=\frac{1}{3} \operatorname{Tr}(\boldsymbol{\epsilon}) \boldsymbol{G}+\boldsymbol{e} ; \boldsymbol{\epsilon}^{*}=\frac{1}{3} \operatorname{Tr}\left(\boldsymbol{\epsilon}^{*}\right) \boldsymbol{G}+\boldsymbol{e}^{*} ; \\
\boldsymbol{\epsilon}^{t h}=\alpha\left(T-T_{i}\right) \boldsymbol{G} ; \boldsymbol{\epsilon}^{p c}=\boldsymbol{e}^{p c} ; \boldsymbol{\epsilon}^{p z}=-\frac{1}{3} g(z) \boldsymbol{G}+\boldsymbol{e}^{p z} \\
\text { with } g(z)= \begin{cases}\frac{3 \chi}{\zeta} z & \left(1^{\text {st }} \text { model }\right) \\
\frac{z}{\zeta} & \left(2^{n d} \text { model }\right)\end{cases}
\end{gathered}
$$

where $\boldsymbol{G}$ denotes the metric tensor (in the case of any canonical basis, $\boldsymbol{G}=\boldsymbol{I}$ where $\boldsymbol{I}$ is a second-order symmetric identity tensor; see [35]), $\operatorname{Tr}(\star)$ is the trace operator associated with the variable $\star,\left(\boldsymbol{\epsilon}^{e l}, \boldsymbol{\epsilon}^{t h}, \boldsymbol{\epsilon}^{p c}, \boldsymbol{\epsilon}^{p z}\right)$ are the (second-order) elastic, thermal, classical plastic and TRIP-like strain tensors, respectively, $T_{i}$ is the initial temperature, $\alpha$ is the thermal expansion coefficient (with is identical in both phases and temperature independent), $g(z)$ denotes the density change resulting from TRIP-like processes, $\zeta$ is a material parameter characterizing the change in the density occurring during the phase transformations and $\chi$ is a material parameter associated with changes in the density (see [32] for more details).

Assuming that the material under consideration is initially untransformed $\left(z_{i}=0\right.$ and $\left.\boldsymbol{e}_{i}^{p z}=0\right)$ and unplasticized ( $v_{i}=0$ and $\boldsymbol{e}_{i}^{p c}=0$ ), the Helmholtz-free energy potential per unit mass, $\psi$, is decomposed into the terms corresponding to the thermal leaks (the irreversible part), the free energy at constant temperature which can be immediately recovered by an elastic unloading process (the reversible part) and the hardening associated with the classical plasticity and the latent heat of irreversible 
solid-solid phase transformation. The state equations are (see [34]) as follows:

$$
\begin{aligned}
& \psi=-C_{\boldsymbol{\epsilon}} \frac{\left(T-T_{i}\right)^{2}}{2 T_{i}}+\frac{1}{2 \rho_{i}} \frac{3 \lambda+2 \mu}{3}[\operatorname{Tr}(\boldsymbol{\epsilon})+g(z)]^{2} \\
& +\frac{\mu}{\rho_{i}}\left[\left(\boldsymbol{e}-\boldsymbol{e}^{p c}-\boldsymbol{e}^{p z}\right):\left(\boldsymbol{e}-\boldsymbol{e}^{p c}-\boldsymbol{e}^{p z}\right)\right]+\frac{1}{2 \rho_{i}} h v^{2} \\
& -\frac{1}{\rho_{i}}(3 \lambda+2 \mu) \alpha\left(T-T_{i}\right)[\operatorname{Tr}(\boldsymbol{\epsilon})+g(z)] \\
& +\frac{\vartheta M}{\rho_{i}}\left[\frac{T}{2} z^{2}-\left(T-T_{i}^{z}\right) z\right]+\psi_{i} \\
& S=-\frac{\partial \psi}{\partial T} \\
& \boldsymbol{\sigma}=-P \boldsymbol{G}+\boldsymbol{s} \\
& P=-\rho \frac{\partial \psi}{\partial \operatorname{Tr}(\boldsymbol{\epsilon})}=-\frac{\rho}{\rho_{i}} \frac{(3 \lambda+2 \mu)}{3}\left[\operatorname{Tr}(\boldsymbol{\epsilon})+g(z)-3 \alpha\left(T-T_{i}\right)\right] \\
& \boldsymbol{s}=\rho \frac{\partial \psi}{\partial \boldsymbol{e}}=\frac{\rho}{\rho_{i}} 2 \mu\left(\boldsymbol{e}-\boldsymbol{e}^{p z}-\boldsymbol{e}^{p c}\right) \\
& \Upsilon^{m}=-\frac{\rho}{\rho_{i}} \frac{\partial \psi}{\partial m}
\end{aligned}
$$

where $S$ denotes the local specific entropy, $\Upsilon^{m}$ denotes the "thermodynamic forces" (so-called stress-like variables) associated with the variables $m=\left(\boldsymbol{e}^{p c}, \boldsymbol{e}^{p z}, v, z\right), \boldsymbol{\sigma}$ is the Cauchy stress tensor, $-P$ and $\boldsymbol{s}$ are the spherical and deviatoric parts of the (second-order symmetric) Cauchy stress-tensor $\boldsymbol{\sigma}$, respectively, $C_{\epsilon}, \mu$ and $\lambda$ are the specific heat capacity and Lamé constants, respectively (which are identical in both phases and independent of the temperature $T), \frac{\partial \dagger}{\partial \star}$ denotes the first partial derivative of $\dagger$ with respect to the variable $\star$, “:” represents the double inner product, $T_{i}^{z}$ is the solid-solid phase transformation temperature, $h$ is a material parameter characterizing the linear isotropic hardening associated with classical plasticity, $\vartheta$ and $M$ are material parameters associated with the solid-solid phase transformation and $\rho_{i} \cong \rho$ and $\psi_{i}$ are both the initial density and Helmholtz-free energy of the material per unit mass.

\subsection{Yield functions and equations of evolution}

In order to define the equations of evolution of the internal variable associated with TRIP-like processes $\left(z\right.$ and $\left.\boldsymbol{e}^{p z}\right)$ and classical plasticity processes ( $v$ and $\boldsymbol{e}^{p c}$ ), two yield functions $f^{p z}$ and $f^{p c}$, which depend on the temperature, stress and internal variables, are introduced. Following the classical rate-dependent approaches (such as those used in viscoplasticity or viscodamage, for example), the yield functions account for the evolution of the dissipative processes (i.e. $f^{p z}>0$ and/or $f^{p c}>0$ ) and can be used to define the resulting elastic domain in terms of $f^{p z} \leq 0$ and $f^{p c} \leq 0$ (where the coupled relations $f^{p z}=0$ and $f^{p c}=0$ represent the boundary of elastic domain; see $[33,34])$.

The two yield functions associated with both TRIPlike $f^{p z}$ and classical plasticity $f^{p c}$ processes are defined as follows (see [33]):

$$
\begin{aligned}
& f^{p z}= \begin{cases}\sigma_{e q}+3 \chi P-\left(\sigma_{y z}(T)+\zeta \beta z\right) & \left(1^{s t} \text { model }\right) \\
\frac{T}{T_{i}^{z}}-\exp \left(-\frac{\langle P\rangle^{+}}{\omega}\right) & \left(2^{\text {nd }} \text { model }\right)\end{cases} \\
& f^{p c}=\sigma_{e q}-\left(\sigma_{y p}+h v\right)
\end{aligned}
$$

where $\sigma_{y z}(T)=\exp \left(\frac{\left(T_{i}-T\right)}{T_{i}}\right) \bar{\sigma}_{i}$ is the TRIP-like yield stress (with $\sigma_{y z}(T)=\bar{\sigma}_{i}$ when $T=T_{i}$ ), $\beta$ is a material parameter associated with the hardening process, $\omega$ is a material parameter characterizing a "pressure sensitivity" level (see [32] for more details), $\sigma_{e q}=\left(\frac{3}{2} s: s\right)^{\frac{1}{2}}$ is the Von Mises equivalent stress and $\sigma_{y p}$ is the classical yield stress and $\langle.\rangle^{+}$are the Macaulay brackets $\left(\langle x\rangle^{+}=x\right.$ when $x \geq 0$ and $\langle x\rangle^{+}=0$ when $x<0$ ).

In the case of viscoinelastic case (which is here plastic type, i.e. viscoplastic), the equations of evolution are:

$$
\begin{aligned}
& \dot{\boldsymbol{e}}^{p c}=\dot{v} \boldsymbol{n}^{p c}=\dot{v} \frac{3}{2 \sigma_{e q}} \boldsymbol{s} \\
& \dot{\boldsymbol{e}}^{p z}=\dot{v} \boldsymbol{n}^{p z}=\dot{p} \frac{3}{2 \sigma_{e q}} \boldsymbol{S} \\
& \dot{z}=\zeta \dot{p}
\end{aligned}
$$

where “ $\dot{*}=\frac{\partial *}{\partial t}$ ” denotes the total time derivative associated with the variable $*, \boldsymbol{n}^{p c}$ and $\dot{v}$ (resp. $\boldsymbol{n}^{p z}$ and $\dot{p}$ ) are the flow direction tensors and viscoinelastic multipliers (so-called inelastic Lagrange multipliers) associated with the classical viscoplasticity (resp. TRIP-like) process which are defined as: 


$$
\begin{aligned}
& \dot{v}=\frac{\left\langle\left. f^{p c}(\boldsymbol{\sigma}, v)\right|^{+}\right.}{\xi \sigma_{y p}} \\
& \dot{p}=\left\{\begin{array}{c}
\frac{\left\langle f^{p z}(\boldsymbol{\sigma}, z ; T)\right\rangle^{+}}{\eta \bar{\sigma}_{i}} H(1-z) H\left(\sigma_{e q}+3 \chi P\right. \\
\left.+\vartheta M\left[(1-z) T-T_{i}^{z}\right]\right)\left(1^{s t} \text { model }\right) \\
\frac{\langle 1-z\rangle^{+}}{\eta}\left\langle f^{p z}(T, P)\right\rangle^{+} H\left(\sigma_{e q}+P\right. \\
\left.+9 M\left[(1-z) T-T_{i}^{z}\right]\right) \quad\left(2^{\text {nd }} \text { model }\right)
\end{array}\right.
\end{aligned}
$$

where $\xi$ (resp. $\eta$ ) denotes the characteristic time of the viscous effects associated with the classical plasticity (resp. TRIP-like) process and $H($.$) is Heaviside step function$ (with $H(x)=1$ when $x \geq 0$ and $H(x)=0$ when $x<0$ ); see Appendix 1.2 for some additional comments.

\section{Constitutive models: discrete framework}

\subsection{Integration scheme: Return mapping algorithm (RMA)}

The constitutive equations involved in two models presented in this study (see Section 2) are implemented numerically using a RMA, [1-4, 36-38]. The following notation is used for this purpose:

- the quantities denoted by $(\star)_{n+1}^{(k)}$ correspond to the $(k)$ th equilibrium iteration at time $t_{n+1}$.

- the quantities denoted by $(\star)_{n}$ are known at time $t_{n}$. Moreover, the quantities not corresponding to $(\star)^{(k)}$ are assumed to be given by the previous equilibrium computation step.

- the incremental finite step $\Delta(\star)_{n+1}^{(k)}$ gives the quantity " $\star$ " between time $t_{n}$ and the $(k)$ th equilibrium iteration at time $t_{n+1}$, i.e. $\Delta(\star)_{n+1}^{(k)}=(\star)_{n+1}^{(k)}-(\star)_{n}$.

When the evolution problem is addressed in terms of discrete incremental approach with a time step $\Delta t_{n+1}$, the thermomechanical constitutive equations reduce to a rule which yields $\boldsymbol{\sigma}_{n+1}^{(k)}$, where the mechanical state $\mathcal{S}_{n}=$ $\left(T_{n}, \operatorname{Tr}\left(\boldsymbol{\epsilon}_{n}\right), e_{n}, \boldsymbol{e}_{n}^{p z}, \boldsymbol{e}_{n}^{p c}, z_{n}, v_{n}, \boldsymbol{\sigma}_{n}\right)$ at time $t_{n}$ and the temperature increment $\Delta T_{n+1}$ (or temperature $T_{n+1}$ at time $t_{n+1}$ ) are known and for any given strain increment $\Delta \boldsymbol{\epsilon}_{n+1}^{(k)}$ such as:

$$
\boldsymbol{\sigma}_{n+1}^{(k)}=\mathbf{R}\left(\mathcal{S}_{n}, \Delta \boldsymbol{\epsilon}_{n+1}^{(k)} ; T_{n+1}\right)
$$

where $\mathbf{R}$ denotes the use of RMA (see [4, 39]).

The main steps in the RMA procedure applied to two thermomechanical models presented here can be summarized as follows: (i) The "trial" thermoelastic stress tensor $\boldsymbol{\sigma}_{n+1}^{e l,(k)}$ which is decomposed into spherical and deviatoric parts, $P_{n+1}^{e l,(k)}$ and $\boldsymbol{s}_{n+1}^{e l,(k)}$, respectively (see eqs. (2)), are calculated as follows:

$$
\begin{gathered}
\boldsymbol{\sigma}_{n+1}^{e l,(k)}=-P_{n+1}^{e l,(k)} \boldsymbol{G}+\boldsymbol{s}_{n+1}^{e l,(k)} \\
\text { with } P_{n+1}^{e l,(k)}=-\left[\frac{1}{3} \operatorname{Tr}\left(\boldsymbol{\sigma}_{n}\right)+\kappa \operatorname{Tr}\left(\Delta \boldsymbol{\epsilon}_{n+1}^{(k)}\right)-3 \kappa \alpha \Delta T_{n+1}\right] \\
\text { and } \boldsymbol{s}_{n+1}^{e l,(k)}=\boldsymbol{s}_{n}+2 \mu \Delta \boldsymbol{e}_{n+1}^{(k)}
\end{gathered}
$$

where $\boldsymbol{s}_{n}=\boldsymbol{\sigma}_{n}-\frac{1}{3} \operatorname{Tr}\left(\boldsymbol{\sigma}_{n}\right) \boldsymbol{G}\left(\right.$ resp. $\left.\boldsymbol{e}_{n}=\boldsymbol{\epsilon}_{n}-\frac{1}{3} \operatorname{Tr}\left(\boldsymbol{\epsilon}_{n}\right) \boldsymbol{G}\right)$ is the stress (resp. strain) deviator tensor at time $t_{n}$ and $\kappa=\frac{(3 \lambda+2 \mu)}{3}$ is the bulk modulus.

(ii) The following two yield criteria (see eq. (3)) are tested (see Figure 2 and also [39]):

1. If $f^{p z}\left(\boldsymbol{\sigma}_{n+1}^{e l,(k)}, p_{n} ; T_{n+1}\right) \leq 0$ (resp. $\left.f^{p z}\left(T_{n+1}, P_{n+1}^{e l,(k)}\right) \leq 0\right)$ in the first (resp. second) model and $f^{p c}\left(\boldsymbol{\sigma}_{n+1}^{e l,(k)}, v_{n}\right) \leq 0$, then the increment will be purely elastic, i.e. $\Delta p_{n+1}^{(k)}=0$, $\Delta v_{n+1}^{(k)}=0$ and we then go directly to [(iv)-(a)].

2. If $f^{p z}\left(\boldsymbol{\sigma}_{n+1}^{e l,(k)}, p_{n} ; T_{n+1}\right)>0$ (resp. $\left.f^{p z}\left(T_{n+1}, P_{n+1}^{e l,(k)}\right)>0\right)$ in the first (resp. second) model and $f^{p c}\left(\boldsymbol{\sigma}_{n+1}^{e l,(k)}, v_{n}\right) \leq 0$, then the increment will be a "TRIP-like" one (due only to solid-solid phase transformation), i.e. $\Delta p_{n+1}^{(k)}>0$, $\Delta v_{n+1}^{(k)}=0$ and we then go to [(iii)-(a)-(1)] (resp. [(iii)(b)-(1)]) in the case of the first (resp. second) model.

3. If $f^{p z}\left(\boldsymbol{\sigma}_{n+1}^{e l,(k)}, p_{n} ; T_{n+1}\right)>0$ (resp. $\left.f^{p z}\left(T_{n+1}, P_{n+1}^{e l,(k)}\right)>0\right)$ in the first (resp. second) model and $f^{p c}\left(\boldsymbol{\sigma}_{n+1}^{e l,(k)}, v_{n}\right)>0$, then the increment will be both a "TRIP-like" and a classical thermoviscoplastic one (due to solid-solid phase transformation and classical plasticity), i.e. $\Delta p_{n+1}^{(k)}>0, \Delta v_{n+1}^{(k)}>0$ and we then go to [(iii)-(a)-(2)] (resp. [(iii)-(b)-(2)]) in the case of the first (resp. second) model.

4. If $f^{p z}\left(\boldsymbol{\sigma}_{n+1}^{e l,(k)}, p_{n} ; T_{n+1}\right) \leq 0$ (resp. $\left.f^{p z}\left(T_{n+1}, P_{n+1}^{e l,(k)}\right) \leq 0\right)$ in the first (resp. second) model and $f^{p c}\left(\boldsymbol{\sigma}_{n+1}^{e l,(k)}, v_{n}\right)>0$, then the increment will be a classical thermoviscoplastic one (resulting only from classical plasticity), i.e. $\Delta p_{n+1}^{(k)}=0, \Delta v_{n+1}^{(k)}>0$ and then we go to [(iii)-(a)-(3)] (resp. [(iii)-(b)-(3)]) in the case of the first model (resp. second model).

(iii) Based on eq. (5), $\Delta p_{n+1}^{(k)} \geq 0$ and $\Delta v_{n+1}^{(k)} \geq 0$ are computed as follows: (i.e. for [(ii)-(2,3,4)]; see Figure 2 and also [39]):

(a) With the first model: 


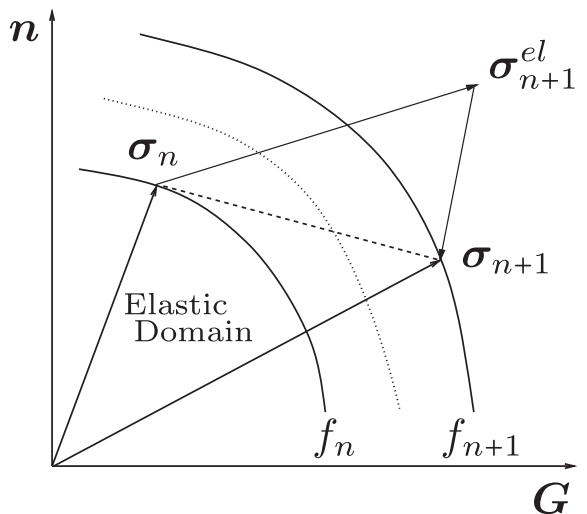

(a)

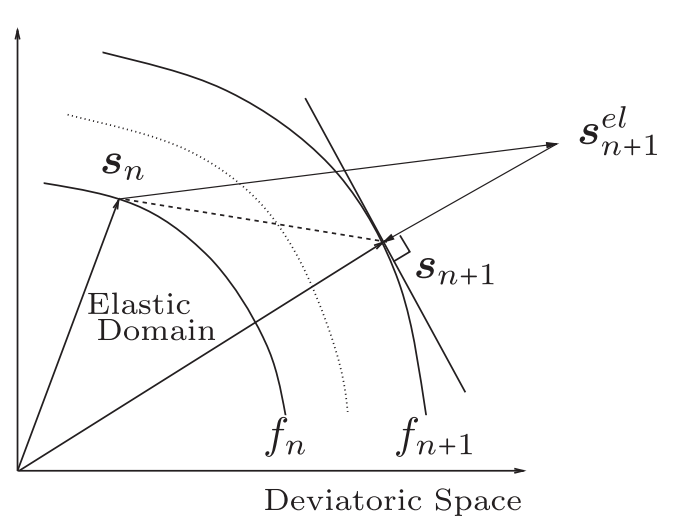

(b)

Figure 1: Geometrical interpretation of RMA: (a) using a pressure-dependent elasto(visco)plastic model in the (G, $\boldsymbol{n}$ )-plane (where $\boldsymbol{n}=\frac{\boldsymbol{s}}{\|\boldsymbol{s}\|}=\sqrt{\frac{3}{2}} \frac{\boldsymbol{s}}{\sigma_{e q}}$ is the unit normal vector in the deviatoric space with respect to the yield surface and $\|\star\|$ is Euclidean norm); (b) using a classical elasto(visco)plastic model (with isochoric plasticity in the deviatoric space; see [39]).

(1) in the case of a "TRIP-like" iteration (corresponding only to the TRIP-like processes) (i.e. for [(ii)-(2)]):

$$
\Delta p_{n+1}^{(k)}=\frac{f^{p z}\left(\boldsymbol{\sigma}_{n+1}^{e l,(k)}, p_{n} ; T_{n+1}\right)}{3 \mu+9 \kappa \chi^{2}+\zeta^{2} \beta+\frac{\eta \bar{\sigma}_{i}}{\Delta t_{n+1}}} ; \Delta v_{n+1}^{(k)}=0
$$

and we then go to [(iv)-(b)]

(2) in the case of a "TRIP-like" and classical thermoviscoplastic iteration (corresponding to the TRIP-like processes and classical plasticity) (i.e. for [(ii)-(3)]):

$$
\begin{aligned}
& \Delta p_{n+1}^{(k)}=\frac{\tilde{B} \tilde{C}_{n+1}^{(k)}-\tilde{A} \tilde{E}_{n+1}^{(k)}}{\tilde{A}\left(\tilde{B}-\frac{\tilde{A} \tilde{D}}{\tilde{B}}\right)}-\frac{\tilde{C}_{n+1}^{(k)}}{\tilde{A}} \\
& \Delta v_{n+1}^{(k)}=\frac{\frac{\tilde{A} \tilde{E}_{n+1}^{(k)}}{\tilde{B}}-\tilde{C}_{n+1}^{(k)}}{\tilde{B}-\frac{\tilde{A} \tilde{D}}{\tilde{B}}}
\end{aligned}
$$

where

$$
\begin{gathered}
\tilde{A}=-3 \mu-9 \kappa \chi^{2}-\zeta^{2} \beta-\frac{\bar{\sigma}_{i} \eta}{\Delta t_{n+1}} ; \tilde{B}=-3 \mu ; \\
\tilde{C}_{n+1}^{(k)}=f^{p z}\left(\boldsymbol{\sigma}_{n+1}^{e l,(k)}, p_{n} ; T_{n+1}\right) ; \tilde{D}=-3 \mu-h-\frac{\sigma_{y p} \xi}{\Delta t_{n+1}} \\
\tilde{E}_{n+1}^{(k)}=f^{p c}\left(\boldsymbol{\sigma}_{n+1}^{e l,(k)}, v_{n}\right)
\end{gathered}
$$

and we then go to [(iv)-(b)]
(3) in the case of a classical thermoviscoplastic iteration (corresponding only to classical plasticity) (i.e. for [(ii)-(4)]):

$$
\Delta v_{n+1}^{(k)}=\frac{f^{p c}\left(\boldsymbol{\sigma}_{n+1}^{e l,(k)}, v_{n}\right)}{3 \mu+h+\frac{\sigma_{y p} \xi}{\Delta t_{n+1}}} ; \Delta p_{n+1}^{(k)}=0
$$

and we then go to [(iv)-(b)]

(b) With the second model:

(1) in the case of a "TRIP-like" iteration (corresponding only to the TRIP-like processes) (for [(ii)-(2)]):

$$
\begin{gathered}
\Delta p_{n+1}^{(k)}=\Delta t_{n+1} \frac{\left\langle 1-z_{n}\right\rangle^{+}}{\eta}\left(\frac{T_{n+1}}{T_{i}^{z}}-\exp \left(-\frac{\left\langle P_{n}\right\rangle^{+}}{\omega}\right)\right) \\
H\left(P_{n}\right) \Delta v_{n+1}^{(k)}=0
\end{gathered}
$$

and we then go to [(iv)-(b)]

(2) in the case of a "TRIP-like" and classical thermoviscoplastic iteration (corresponding to the TRIP-like processes and classical plasticity) (for [(ii)-(2)]):

$$
\begin{gathered}
\Delta p_{n+1}^{(k)}=\Delta t_{n+1} \frac{\left\langle 1-z_{n}\right\rangle^{+}}{\eta}\left(\frac{T_{n+1}}{T_{i}^{z}}-\exp \left(-\frac{\left\langle P_{n}\right\rangle^{+}}{\omega}\right)\right) \\
H\left(P_{n}\right) \Delta v_{n+1}^{(k)}=\frac{f^{p c}\left(\boldsymbol{\sigma}_{n+1}^{e l,(k)}, v_{n}\right)}{3 \mu+h+\frac{\sigma_{y p} \xi}{\Delta t_{n+1}}}
\end{gathered}
$$

and we then go to [(iv)-(b)]

(3) in the case of a classical thermoviscoplastic iteration (corresponding only to classical plasticity) (for [(ii)-(3)]): 


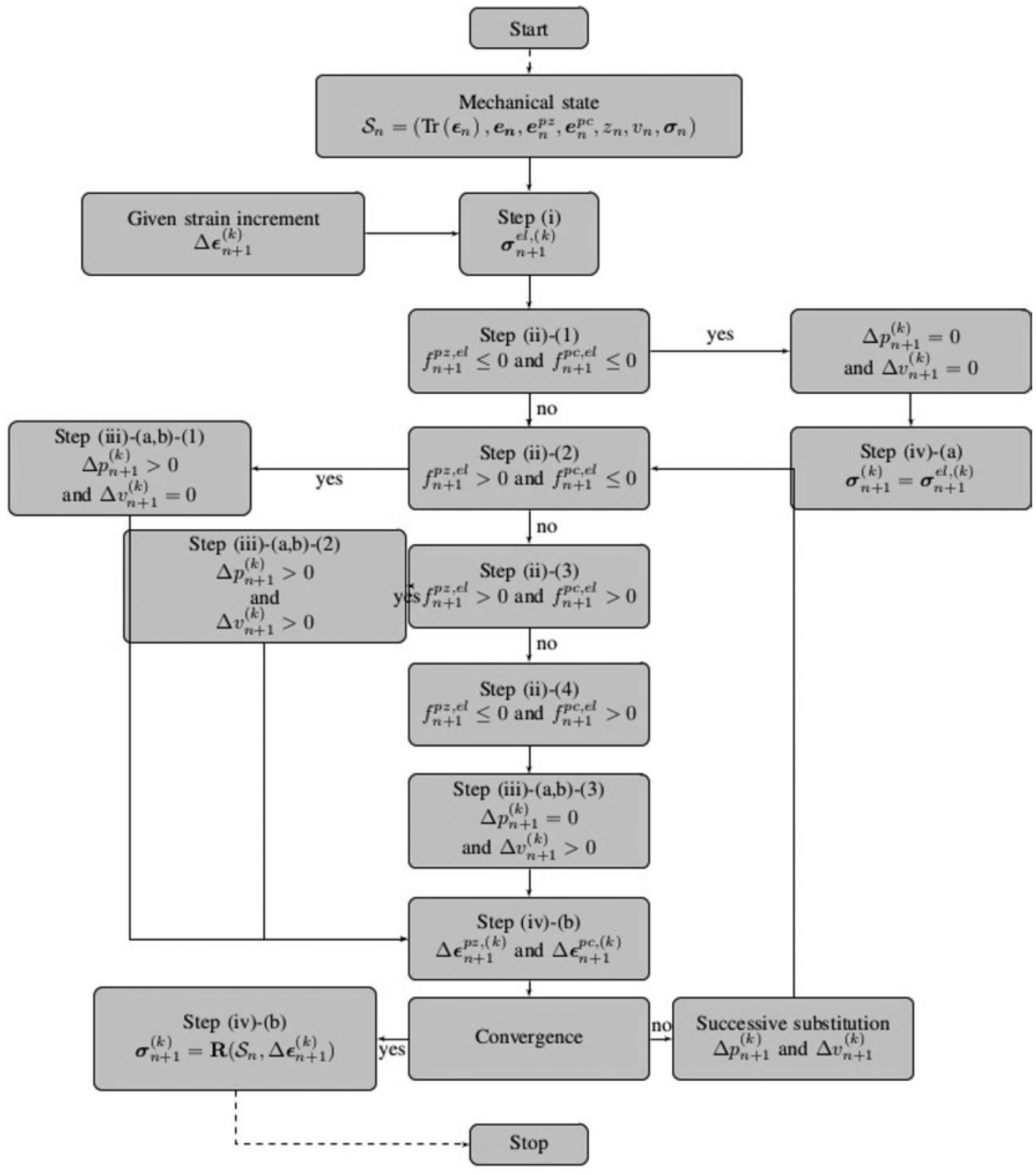

Figure 2: Flowchart for the numerical integration of the constitutive equations involved in two models.

$$
\Delta v_{n+1}^{(k)}=\frac{f^{p c}\left(\boldsymbol{\sigma}_{n+1}^{e l,(k)}, v_{n}\right)}{3 \mu+h+\frac{\sigma_{y p} \xi}{\Delta t_{n+1}}} ; \Delta p_{n+1}^{(k)}=0
$$

and we then go to [(iv)-(b)]

(iv) Updating the mechanical state $\mathcal{S}_{n+1}$ (see eqs. (2) and (4)- (5)) gives (see Figure 2 and also [39]):

(a) in the case of a thermoelastic iteration (i.e. for [(ii)-(a)-(1)] and [(ii)-(b)-(1)]):

$$
\begin{gathered}
\boldsymbol{\sigma}_{n+1}^{(k)}=\boldsymbol{\sigma}_{n+1}^{e l,(k)} ; \boldsymbol{\epsilon}_{n+1}^{p z,(k)}=\boldsymbol{\epsilon}_{n}^{p z} ; \boldsymbol{\epsilon}_{n+1}^{p c,(k)}=\boldsymbol{\epsilon}_{n}^{p c} ; \\
z_{n+1}^{(k)}=z_{n} ; v_{n+1}^{(k)}=v_{n}
\end{gathered}
$$

(b) in the case of a thermoelastoviscoplastic iteration (i.e. for [(iii)-(a)-(2,3,4)] and [(iii)-(b)$(2,3,4)])$ :

$$
\begin{gathered}
\boldsymbol{\sigma}_{n+1}^{(k)}=-\left[P_{n+1}^{e l,(k)}-\kappa g\left(\Delta z_{n+1}^{(k)}\right)\right] \boldsymbol{G}+\boldsymbol{s}_{n+1}^{e l,(k)} \\
-2 \mu\left[\Delta \boldsymbol{\epsilon}_{n+1}^{p z,(k)}+\Delta \boldsymbol{\epsilon}_{n+1}^{p c,(k)}\right] ; \\
\boldsymbol{\epsilon}_{n+1}^{p z,(k)}=\boldsymbol{\epsilon}_{n}^{p z}+\Delta \boldsymbol{\epsilon}_{n+1}^{p z,(k)} ; \boldsymbol{\epsilon}_{n+1}^{p c,(k)}=\boldsymbol{\epsilon}_{n}^{p c}+\Delta \boldsymbol{\epsilon}_{n+1}^{p c,(k)} ; \\
z_{n+1}^{(k)}=z_{n}+\zeta \Delta p_{n+1}^{(k)} ; v_{n+1}^{(k)}=v_{n}+\Delta v_{n+1}^{(k)}
\end{gathered}
$$

with the increment of classical plastic (resp. TRIP-like) strain $\Delta \boldsymbol{\epsilon}_{n+1}^{p c,(k)}$ (resp. $\Delta \boldsymbol{\epsilon}_{n+1}^{p z,(k)}$ ) and the 
increment of mass fraction $\Delta z_{n+1}^{(k)}$ which are given by (see eq. (4); see also Appendix 1.3 for some additional comments):

$$
\begin{gathered}
\Delta \boldsymbol{\epsilon}_{n+1}^{p c,(k)}=\frac{3}{2} \frac{\Delta v_{n+1}^{(k)}}{\left(\sigma_{n+1}^{e l,(k)}\right)_{e q}} \boldsymbol{s}_{n+1}^{e l,(k)} ; \\
\Delta \boldsymbol{\epsilon}_{n+1}^{p z,(k)}=\frac{3}{2} \frac{\Delta p_{n+1}^{(k)}}{\left(\sigma_{n+1}^{e l,(k)}\right)_{e q}} \boldsymbol{s}_{n+1}^{e l,(k)} ; \Delta z_{n+1}^{(k)}=\zeta \Delta p_{n+1}^{(k)}
\end{gathered}
$$

\subsection{The Newton-Raphson method and its variants with a consistent tangent operator}

\subsubsection{Overall equilibrium problem: The Newton-Raphson method}

The classical Newton-Raphson algorithm used to solve the overall equilibrium problem requires the use of a global tangent operator which is also called the "global tangent modulus" or "global tangent stiffness" (Figure 3(a)). In order to ensure a quadratic convergence, the global tangent operator must have as its main feature to be compatible with Newton-Raphson method. Therefore, the local tangent operator, which is determined at each integration point in the structure and then assembled to form the global tangent operator, must also have the property of being "consistent" with the integration scheme used for the local inelastic material behaviour (see [39]). However, in the case of some non-linear structural problems (involving a large number of finite elements), the classical Newton-Raphson method can no longer be envisaged since it involves a prohibitively long computing time due to the factorization of the global stiffness matrix (associated with the tangent operator) which is updated at each loading increment (see [40]). To address this issue, a modified Newton-Raphson method [41] (in which a constant tangent operator, such as the elastic operator, is used) is taken to be an effective solution, and although it does not lead to a quadratic convergence, the calculation time required is much shorter than with the classical method (Figure 3-(b)).

For solving a non-linear problem with the NewtonRaphson procedure (to $(k+1)$ th iteration at time $\left.t_{n+1}\right)$, the overall equilibrium must check:

$$
\begin{aligned}
\left\{\mathcal{R}_{n+1}^{(k+1)}\right\} & =\{0\} \Longleftrightarrow\left[\mathcal{K}_{n+1}^{(k)}\right]\left\{\delta u_{n+1}^{(k+1)}\right\} \\
& =-\left\{\mathcal{R}_{n+1}^{(k)}\right\}=\left\{\mathcal{F}_{n+1}^{e x}\right\}-\left\{\mathcal{F}_{n+1}^{i n,(k)}\right\}
\end{aligned}
$$
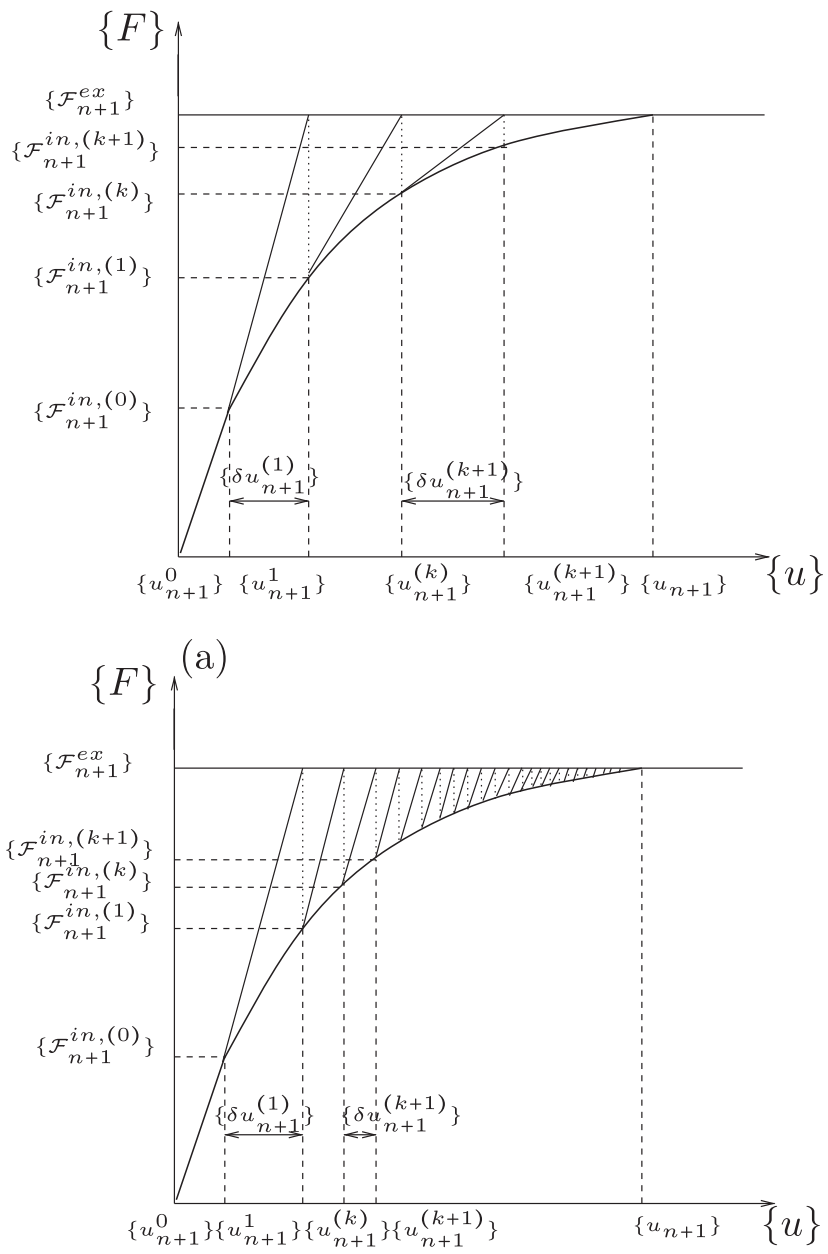

(b)

Figure 3: Geometrical interpretation of the Newton-Raphson method for overall equilibrium problem with (a) exact tangent operator,

(b) constant tangent operator (elastic type; see [33, 39]); Notations: $\left\{u_{n+1}^{0}\right\}=\left\{u_{n}\right\}$ and $\left\{\mathcal{F}_{n+1}^{i n,(0)}\right\}=\left\{\mathcal{F}_{n}^{i n}\right\}$.

where $\{*\}$ (resp. $[*]$ ) denotes a quantity of vector type (resp. matrix type), $\left[\mathcal{K}_{n+1}^{(k)}\right]=\left[\frac{\partial\{\mathcal{R}\}}{\partial\{u\}}\right]_{n+1}^{(k)}=\sum_{j=1}^{N^{e l e m}}\left[\mathcal{K}_{n+1}^{(k)}\right]_{j}$ is the global tangent stiffness matrix to $(k)$ th iteration at time $t_{n+1}$ (with $N^{\text {elem }}$ is the total number of elements in the structure considered), $\left\{\delta u_{n+1}^{(k+1)}\right\}=\left\{u_{n+1}^{(k+1)}\right\}-\left\{u_{n+1}^{(k)}\right\}=$ $\left\{\Delta u_{n+1}^{(k+1)}\right\}-\left\{\Delta u_{n+1}^{(k)}\right\}$ is the (unknown) increment displacement vector to $(k+1)$ th iteration at time $t_{n+1},\left\{\mathcal{F}_{n+1}^{e x}\right\}$ is the vector of external (applied) forces at time $t_{n+1},\left\{\mathcal{F}_{n+1}^{i n,(k)}\right\}$ is the vector of internal forces to $(k)$ th iteration at time $t_{n+1}$ and $\left\{\mathcal{R}_{n+1}^{(k)}\right\}$ (resp. $\left\{\mathcal{R}_{n+1}^{(k+1)}\right\}$ ) is the residual force vector to $(k)$ th (resp. $(k+1)$ th) iteration at time $t_{n+1}$ which characterizes the imbalance of the equilibrium equation (i.e. the overall equilibrium of the structure is reached when the Euclidean norm of the residue equilibrium 
$\left\|\left\{\mathcal{R}_{n+1}^{(k+1)}\right\}\right\|$ is less than a tolerance parameter $p_{T O L}$ such as $\left.\left\|\left\{\mathcal{R}_{n+1}^{(k+1)}\right\}\right\| \leq p_{\text {TOL }}\right)$.

\subsubsection{Local consistent tangent operator (CTO)}

In this section, we propose to develop the local CTO (see Figure 4; for example [2]; [4]; [39]) associated only with the first model $\mathcal{T}_{n+1}^{(k)}\left(\mathcal{S}_{n}, \Delta \boldsymbol{\epsilon}_{n+1}^{(k)} ; T_{n+1}\right)$ (which is a fourthorder tensor; see [32]) at constant temperature $T=T_{n+1}$, i.e.:

$$
\begin{aligned}
\mathcal{T}_{n+1}^{(k)}\left(\mathcal{S}_{n}, \Delta \boldsymbol{\epsilon}_{n+1}^{(k)} ; T_{n+1}\right) & =\left.\frac{\partial \boldsymbol{\sigma}_{n+1}^{(k)}}{\partial \Delta \boldsymbol{\epsilon}_{n+1}^{(k)}}\right|_{T=T_{n+1}} \\
& =\frac{\partial \mathbf{R}\left(\mathcal{S}_{n}, \Delta \boldsymbol{\epsilon}_{n+1}^{(k)} ; T_{n+1}\right)}{\partial \Delta \boldsymbol{\epsilon}_{n+1}^{(k)}}
\end{aligned}
$$

In line with Section 2, the Cauchy stress tensor $\boldsymbol{\sigma}_{n+1}^{(k)}=$ $\mathbf{R}\left(\mathcal{S}_{n}, \Delta \boldsymbol{\epsilon}_{n+1}^{(k)} ; T_{n+1}\right)$ reads as follows:

$$
\begin{aligned}
\boldsymbol{\sigma}_{n+1}^{(k)}= & \boldsymbol{\sigma}_{n}+\mathcal{E}: \Delta \boldsymbol{\epsilon}_{n+1}^{(k)}+\kappa\left[g\left(\Delta z_{n+1}^{(k)}\right)-3 \alpha \Delta T_{n+1}\right] \boldsymbol{G} \\
& -2 \mu\left(\Delta \boldsymbol{e}_{n+1}^{p z,(k)}+\Delta \boldsymbol{e}_{n+1}^{p c,(k)}\right)
\end{aligned}
$$

where $\mathcal{E}=3 \kappa(\boldsymbol{G} \otimes \boldsymbol{G})+2 \mu\left[\boldsymbol{G} \otimes \boldsymbol{G}-\frac{1}{3} \boldsymbol{G} \otimes \boldsymbol{G}\right]$ denotes the elastic stiffness operator (fourth-order tensor associated with Hooke's law) and $\otimes$ (resp. $\otimes$ ) is the classical (resp.

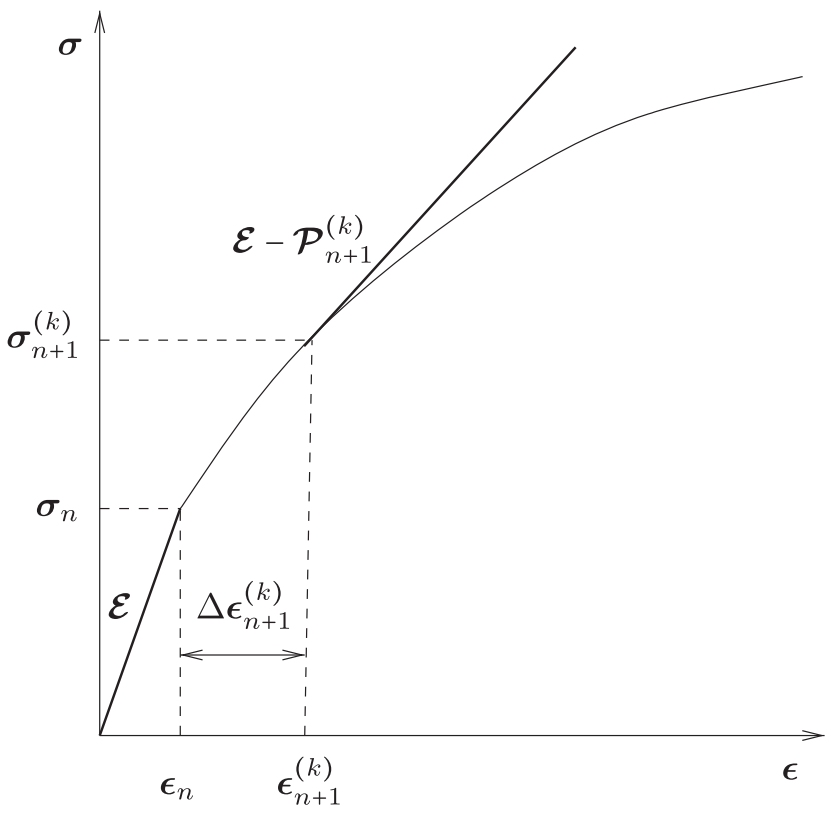

Figure 4: Geometrical interpretation of the local CTO $\mathcal{T}_{n+1}^{(k)}$ (see [39]). non-classical) tensorial product (see [35]) such that in any canonical basis $\left\{\boldsymbol{e}_{i} \otimes \boldsymbol{e}_{j} \otimes \boldsymbol{e}_{k} \otimes \boldsymbol{e}_{l}\right\}$, i.e. $[G \otimes G]_{i j k l}=\delta_{i j} \delta_{k l}$ (resp. $[G \otimes G]_{i j k l}=\frac{1}{2}\left(\delta_{i k} \delta_{j l}+\delta_{j k} \delta_{i l}\right)$ ) (see [39]).

After combining eqs. (18) and (19), the local CTO $\mathcal{T}_{n+1}^{(k)}$ reduces to:

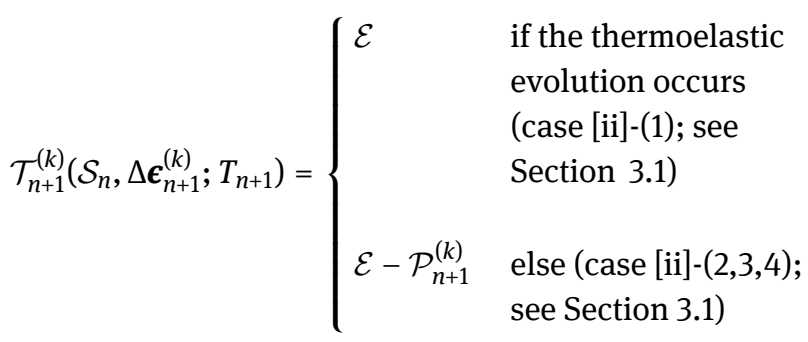

where $\mathcal{P}$ is the operator of "inelastic-corrections" (i.e. fourth-order tensor caused by the irreversible processes; see [39]) which reads:

1. in the case of a "TRIP-like" iteration (corresponding only to TRIP-like processes; case [(ii)-(2)], see Section 3.1):

$$
\begin{aligned}
\mathcal{P}_{n+1}^{(k)} & =3 \mu\left(3 \mu a_{n+1}^{(k)}-b_{n+1}^{(k)}\right)\left(\frac{\boldsymbol{s}_{n+1}^{e l,(k)}}{\left(\sigma_{n+1}^{e l,(k)}\right)_{e q}} \otimes \frac{\boldsymbol{s}_{n+1}^{e l,(k)}}{\left(\sigma_{n+1}^{e l,(k)}\right)_{e q}}\right) \\
& +2 \mu b_{n+1}^{(k)}\left[\boldsymbol{G} \otimes \boldsymbol{G}-\frac{1}{3} \boldsymbol{G} \otimes \boldsymbol{G}\right]+9 \kappa^{2} \chi^{2} a_{n+1}^{(k)}(\boldsymbol{G} \otimes \boldsymbol{G}) \\
& -9 \kappa \mu \chi a_{n+1}^{(k)}\left(\frac{\boldsymbol{s}_{n+1}^{e l,(k)}}{\left(\sigma_{n+1}^{e l,(k)}\right)_{e q}} \otimes \boldsymbol{G}+\boldsymbol{G} \otimes \frac{\boldsymbol{s}_{n+1}^{e l,(k)}}{\left(\sigma_{n+1}^{e l,(k)}\right)_{e q}}\right)
\end{aligned}
$$

with

$$
\begin{aligned}
& a_{n+1}^{(k)}=\frac{1}{\left(3 \mu+9 \kappa \chi^{2}+\zeta^{2} \beta+\frac{\eta \bar{\sigma}_{i}}{\Delta t_{n+1}}\right)} ; \\
& b_{n+1}^{(k)}=3 \mu \frac{\Delta p_{n+1}^{(k)}}{\left(\sigma_{n+1}^{e l,(k)}\right)_{e q}}
\end{aligned}
$$

2. in the case of a "TRIP-like" and classical thermoviscoplastic iteration (corresponding to both TRIP-like and classical plasticity processes; i.e. case [(ii)-(3)], see Section 3.1): 


$$
\begin{aligned}
& \mathcal{P}_{n+1}^{(k)}= \tilde{a}_{n+1}^{(k)}\left(\frac{\boldsymbol{s}_{n+1}^{e l,(k)}}{\left(\sigma_{n+1}^{e l,(k)}\right)_{e q}} \otimes \frac{\boldsymbol{s}_{n+1}^{e l,(k)}}{\left(\sigma_{n+1}^{e l,(k)}\right)_{e q}}\right) \\
&+\tilde{b} {\left[\left(\frac{\boldsymbol{s}_{n+1}^{e l,(k)}}{\left(\sigma_{n+1}^{e l,(k)}\right)_{e q}} \otimes \boldsymbol{G}\right)+\left(\boldsymbol{G} \otimes \frac{\boldsymbol{s}_{n+1}^{e l,(k)}}{\left(\sigma_{n+1}^{e l,(k)}\right)_{e q}}\right)\right] } \\
&+\tilde{c}_{n+1}^{(k)}\left[\boldsymbol{G} \otimes \boldsymbol{G}-\frac{1}{3} \boldsymbol{G} \otimes \boldsymbol{G}\right]+\tilde{d} \boldsymbol{G} \otimes \boldsymbol{G}
\end{aligned}
$$

with

$$
\begin{aligned}
\tilde{a}_{n+1}^{(k)}= & 3 \mu\left[\frac{3 \mu\left(\frac{\tilde{A}}{\tilde{B}}-1\right)}{\left(\tilde{B}-\frac{\tilde{A} \tilde{D}}{\tilde{B}}\right)}-\frac{2 \Delta p_{n+1}^{(k)}}{\left(\sigma_{n+1}^{e l,(k)}\right)_{e q}}\right. \\
& \left.+\frac{3 \mu(\tilde{B}-\tilde{A})}{\tilde{A}\left(\tilde{B}-\frac{\tilde{A} \tilde{D}}{\tilde{B}}\right)}-\frac{3 \mu}{\tilde{A}}\right] ; \\
\tilde{b}= & \frac{9 \kappa \mu}{\tilde{A}}\left[\frac{\tilde{B}-\tilde{A}}{\left.\tilde{B}-\frac{\tilde{A} \tilde{D}}{\tilde{B}}-1\right) ; \tilde{c}_{n+1}^{(k)}=12 \mu^{2} \frac{\Delta p_{n+1}^{(k)}}{\left(\sigma_{n+1}^{e l,(k)}\right)_{e q}} ;}\right. \\
\tilde{d}= & \frac{(3 \kappa)^{2} \chi^{2}}{\tilde{A}}\left[\frac{\tilde{B}}{\tilde{B}-\frac{\tilde{A} \tilde{D}}{\tilde{B}}}-1\right]
\end{aligned}
$$

and

$$
\begin{aligned}
& \tilde{A}=-3 \mu-9 \kappa \chi^{2}-\zeta^{2} \beta-\frac{\bar{\sigma}_{i} \eta}{\Delta t_{n+1}} ; \tilde{B}=-3 \mu ; \\
& \tilde{D}=-3 \mu-h-\frac{\sigma_{y p} \xi}{\Delta t_{n+1}}
\end{aligned}
$$

3. in the case of a classical thermoviscoplastic iteration (corresponding only to the framework of classical plasticity; i.e. case [(ii)-(4)], see Section 3.1):

$$
\begin{aligned}
\mathcal{P}_{n+1}^{(k)} & =3 \mu\left(3 \mu \tilde{a}-\tilde{b}_{n+1}^{(k)}\right)\left(\frac{\boldsymbol{s}_{n+1}^{e l,(k)}}{\left(\sigma_{n+1}^{e l,(k)}\right)_{e q}} \otimes \frac{\boldsymbol{s}_{n+1}^{e l,(k)}}{\left(\sigma_{n+1}^{e l,(k)}\right)_{e q}}\right) \\
& +2 \mu \tilde{b}_{n+1}^{(k)}\left[\boldsymbol{G} \otimes \boldsymbol{G}-\frac{1}{3} \boldsymbol{G} \otimes \boldsymbol{G}\right]
\end{aligned}
$$

with

$$
\tilde{a}=\frac{1}{\left(3 \mu+h+\frac{\sigma_{y p} \xi}{\Delta t_{n+1}}\right)} ; \tilde{b}_{n+1}^{(k)}=3 \mu \frac{\Delta v_{n+1}^{(k)}}{\left(\sigma_{n+1}^{e l,(k)}\right)_{e q}}
$$

\section{Numerical examples}

Using the numerical procedure presented in Section 3, these models have been implemented in the finiteelement software Code_Aster (which is developed by French nuclear power company known as EDF Group). This section is devoted to test, assess and discuss both the predictive abilities associated with RMA numerical procedure involving two plasticity type processes (see Section 2) on some numerical examples.

\subsection{Geometry and boundary conditions}

Let $\Omega$ be a two-dimensional material domain (square in shape, measuring $100 \mathrm{~mm}$ on the side) in the $x-y$ plane. The boundary of $\Omega$ denoted by $\partial \Omega$ will be divided into six line segments (see Figure 5-(a)):

$$
\partial \Omega=[A B] \cup[B C] \cup[C D] \cup[D E] \cup[E F] \cup[F A]
$$

where $[B C]$ denotes a part of the upper side of the square, which is $100 \mathrm{~mm}$ in length. In $\Omega$, a finite-element mesh composed of approximately 3000 quadratic TR6 triangular elements is generated with a more highly refined mesh in the vicinity of the line segment $[B C]$ (see Figure 5-(b)) where the loading is applied. This mesh is systematically used in all the numerical simulations presented in this section.

\subsection{Preliminary thermal problem (PB1)}

We consider here a weak thermomechanical coupling with the presented two models (see Section 3), i.e. the temperature field affects unilaterally the mechanical fields. Due to the thermal boundary conditions (see Eq. 26), the temperature field acting in $\Omega$ can therefore be computed without any mechanical considerations.

Neglecting the source terms corresponding to thermoelasticity, classical plasticity and irreversible solidsolid phase transformations and considering the case of steady-state conduction, the heat equation reduces simply to $\operatorname{Div}(\operatorname{Grad}(T))=0$ (with $\operatorname{Div}(\dagger)$ and $\mathbf{G r a d}(\ddagger)$ being the divengence and gradient operators associated with the 
$\sigma_{n} \neq 0$ and $\sigma_{t} \neq 0$ and/or $T \neq T_{i}$

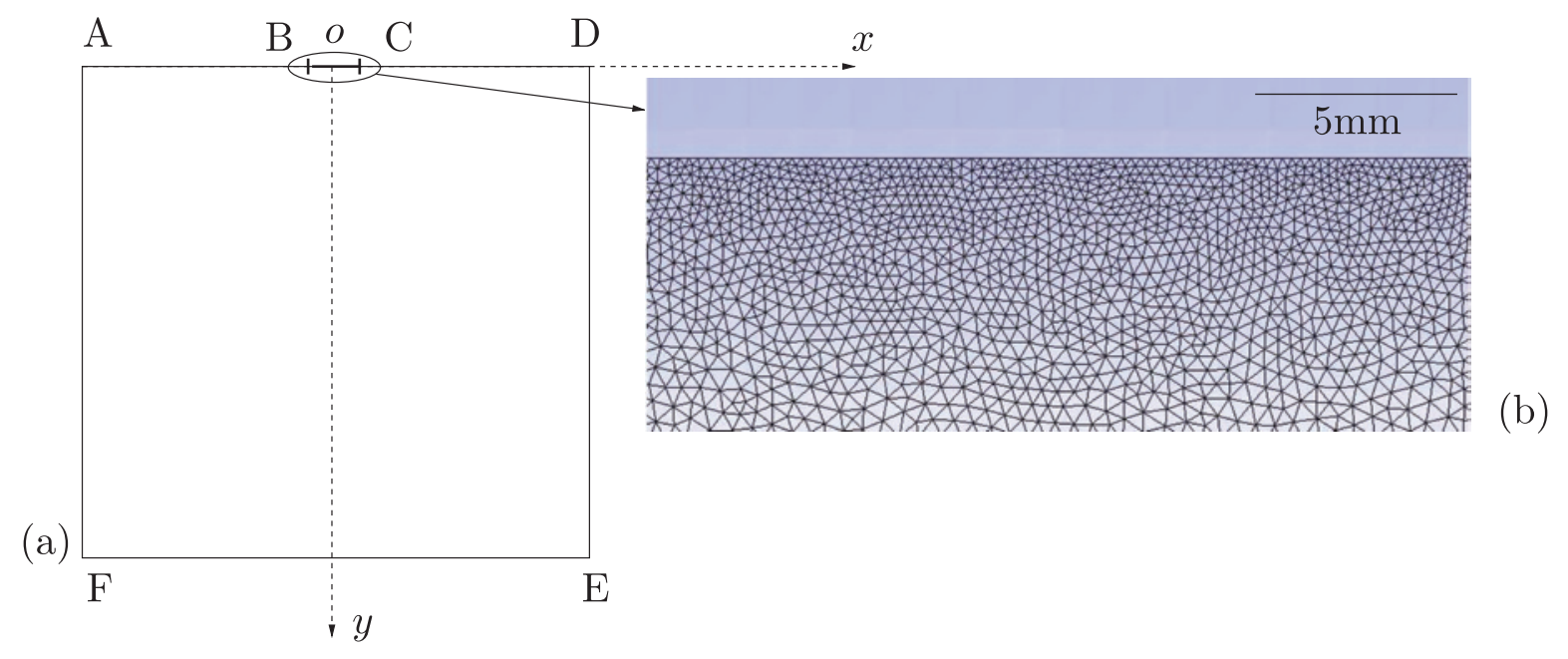

Figure 5: (a) Two-dimensional material domain (square in shape, measuring $100 \mathrm{~mm}$ on each side) in the $x-y$ plane (origin: $o$ ) and boundary conditions pertaining at $[B C]$; (b) Zoom of the refined mesh.

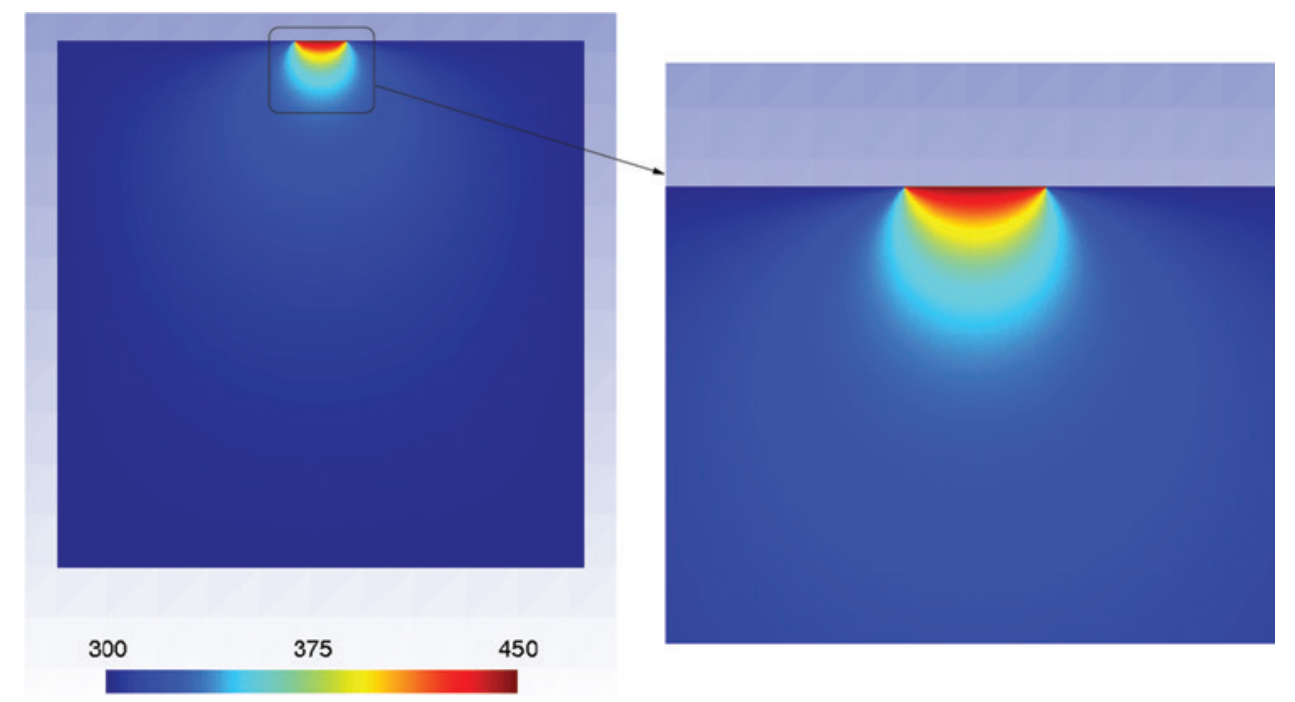

Figure 6: (a) Steady temperature field for thermal problem PB1 (with temperature unit is Kelvin K).

variable $†$ and $\neq$, respectively). Using of standard linear thermal subroutine associated with the finite-element Code_Aster enables to provide the corresponding temperature field which is plotted in Figure 6. When dealing with this purely thermal problem PB1 (where the mechanical boundary conditions do not need to be taken into account in $\partial \Omega$ ), the temperature field is assumed to be a plane field and the thermal boundary conditions are such that:

$$
\begin{aligned}
T & =T_{i}=300 \mathrm{~K} \text { on }[A B] \cup[C D] \cup[D E] \cup[E F] \cup[F A] \\
T & =T^{g}=450 \mathrm{~K} \text { on }[B C]
\end{aligned}
$$

The maximum temperature (i.e. imposed externe temperature $T^{g}$ ) is equal to $450 \mathrm{~K}$ at $[B C]$, whereas the temperature on the other sides of the square remains to the room temperature (i.e. initial temperature $T_{i}$ ) which is $300 \mathrm{~K}$.

\subsection{Thermomechanical problem (PB2)}

In this thermomechanical problem (PB2), the temperature field calculated in the preliminary thermal problem (PB1) (see Section 4.2) is used as an initial data, i.e. the temperature field associated with the subproblem PB1 (see e.g. [42]) noted $T_{0}$ is used here as a known parameter. Therefore, the Cauchy stress tensor $\boldsymbol{\sigma}$ can be written as follows: 


$$
\begin{aligned}
& \boldsymbol{\sigma}=\boldsymbol{\sigma}_{0}+\kappa \operatorname{Tr}(\boldsymbol{\epsilon}) \boldsymbol{G}+2 \mu\left(\boldsymbol{e}-\boldsymbol{e}^{p c}\right) \\
& \text { with } \boldsymbol{\sigma}_{0}=\kappa\left[g\left(z\left(T_{0}\right)\right)-3 \alpha\left(T_{0}-T_{i}\right)\right] \boldsymbol{G}-2 \mu \boldsymbol{e}^{p z}\left(T_{0}\right)
\end{aligned}
$$

where $\boldsymbol{\sigma}_{0}$ denote the thermal prestresses associated with PB1, $z\left(T_{0}\right)$ and $\boldsymbol{e}^{p z}\left(T_{0}\right)$ represent the variable $z$ and $\boldsymbol{e}^{p z}$ which depend on the temperature field $T_{0}$ associated with PB1.

Under the plane strain assumption, the mechanical boundary conditions are as follows (see [34]):

$$
\begin{aligned}
& \boldsymbol{u}=\mathbf{0} \text { on }[E F] ; \boldsymbol{\sigma} \cdot \boldsymbol{n}=\sigma_{n} \boldsymbol{n}+\boldsymbol{\sigma}_{t}=\mathbf{0} \\
& \text { on }[A B] \cup[C D] \cup[D E] \cup[F A] ; \\
& \boldsymbol{\sigma} \cdot \boldsymbol{n}=-\boldsymbol{\sigma} \cdot \boldsymbol{e}_{y}=-\sigma_{n} \boldsymbol{e}_{y}-\left\|\boldsymbol{\sigma}_{t}\right\| \boldsymbol{e}_{x} \text { on }[B C] \\
& \text { with } \sigma_{n}=\dot{P}^{g} t\left(1-\left(\frac{x}{5}\right)^{2}\right)^{\frac{1}{2}} \text { and }\left\|\boldsymbol{\sigma}_{t}\right\|=\mu\left|\sigma_{n}\right| \\
& \text { where } \dot{P}^{g}=100 \mathrm{MPa} \cdot \mathrm{s}^{-1} \text { and } \mu=0.3
\end{aligned}
$$

where $\boldsymbol{\sigma} \cdot \boldsymbol{n}=\sigma_{n} \boldsymbol{n}+\boldsymbol{\sigma}_{t}$ denotes the stress vector, $\sigma_{n}=(\boldsymbol{\sigma} \cdot \boldsymbol{n}) \cdot \boldsymbol{n}$ (resp. $\boldsymbol{\sigma}_{t}$ ) is the normal (resp. tangential) component associated with stress vector, $x$ (resp. $t$ ) is the spatial (resp. time) variable, $\dot{P}^{g}$ is the value of the maximum pressure rate, "." is the simple inner product, $\|\star\|$ is the Euclidean norm, $|*|$ is the absolute-value function $(|x|=x$ when $x<0,|x|=x$ when $x \geq 0)$ and $\left(\boldsymbol{e}_{x}, \boldsymbol{e}_{x}\right)$ is the orthonormal basis. It is important to emphasize that (i) an additional relation between normal and tangential stresses is considered here i.e. $\left\|\boldsymbol{\sigma}_{t}\right\|=\mu\left|\sigma_{n}\right|$ where $\mu$ is a parameter; (ii) it can be deduced from eq. (28) that the maximum of pressure $\sigma_{n}=P^{g}$ is reached in the centre of [BC] (i.e. when $x=0$ then $P^{g}=1000 \mathrm{MPa}$ at time $\left.t=10 \mathrm{~s}\right)$ and the minimum is achieved on the edge of $[B C]$ (i.e. when $x= \pm 5 \mathrm{~mm}$ then $P^{g}=0 \mathrm{MPa}$ at time $t=10 \mathrm{~s}$ ).

The materials simulated in the two models have the following mechanical parameters in common (corresponding to a standard steel type; see [34]):

$$
\begin{aligned}
& \lambda=115 \times 10^{3} \mathrm{MPa} ; \mu=77 \times 10^{3} \mathrm{MPa} ; \\
& \rho_{i}=78 \times 10^{-7} \mathrm{~kg} \cdot \mathrm{mm}^{-3} ; \\
& \alpha=12.10^{-6} \mathrm{~K}^{-1} ; \sigma_{y p}=400 \mathrm{MPa} ; h=2 \times 10^{4} \mathrm{MPa} ; \\
& \xi=2.5 \times 10^{2} \mathrm{~s} ; \delta=0
\end{aligned}
$$

On the other hand, the specific mechanical parameters to each model are (see [32]):

$$
\begin{aligned}
& \chi=\frac{1}{3} ; \bar{\sigma}_{i}=75 \mathrm{MPa} ; \zeta=10^{2} ; \beta=5 \mathrm{MPa} ; \\
& \eta=13 \times 10^{2} \mathrm{~s} ; \quad \text { (first model) } \\
& T_{i}^{z}=1000 \mathrm{~K} ; \omega=700 \mathrm{MPa} ; \zeta=10^{2} \text {; } \\
& \eta=10^{3} \mathrm{~s} \quad \text { (second model) }
\end{aligned}
$$

\subsection{Discussion and results}

The $z$-field at time $t=10 \mathrm{~s}$, when $P(x=0)=1000 \mathrm{MPa}$, is plotted in Figure 7-(a) (resp. Figure 7-(b)) in the case of the first (resp. second) model. Note that with the first model (see Figure 7-(a), a complete transformation is obtained (i.e. $z=1$ ) in the region where the thermomechanical loading is applied. The $z$-field observed in Figure 7-(a), along the $o-y$ axis, is extended down to a depth of about $5 \mathrm{~mm}, z=z_{\max }=1$ which shows that the material is fully transformed (only one phase is present in this region). However, at a depth of $5<y \leq 5 \mathrm{~mm}$, a mixed zone occurs, where the parent and daughter phases are both present. Beyond this depth, at around $y \approx 50 \mathrm{~mm}$, only the initial, untransformed material (i.e. $z=0$ ) could be observed. In the case of the second model (see Figure 7-(b)), the transformation occurs in the immediate vicinity of the surface $[B C]$ where the thermomechanical loading is applied. The $z$-field observed in Figure 7-(b) is quite non-uniform, on the $o-y$ axis such as: (i) $z=z_{\max } \approx 28 \times 10^{-2}$ when $y=0$, which corresponds only to a partial transformation occurring at the place where the thermomechanical loading is applied; (ii) $z=13.10^{-2}$ when $y \approx 35 \times 10^{-1} \mathrm{~mm}$. Beyond a depth of around $6 \mathrm{~mm}$, which is the maximum depth transformed, the transformation is zero (i.e. $z=z_{\min }=0$ ) and therefore the material is still in the initial state.

The $v$-field obtained with the first and second models at time $t=10 \mathrm{~s}$ is plotted in Figure 8-(a) and Figure 8-(b). It is worth noting that, in line with the numerical implementation (see Section 3), the results obtained with the models show that TRIP-like and classical plasticity processes are highly correlated. As can be seen from Figure 8-(a), the first model gives $v=v_{\max } \approx 62 \times 10^{-4}$ on $[B C]$, where the thermomechanical loading is applied. Concerning the second model (Figure 8-(b)), $v=v_{\min }=0$ at $[B C]$ and a maximum value $v_{\max }$ is reached around $21 \times 10^{-3}$ in $\Omega$.

In short, these results show that the first model can predict the occurrence of a complete "quasi-uniform" phase transformation (i.e. $z=1$ ) near the surface where the thermomechanical loading is applied. The second model is able to predict a transformed region which has a smaller depth than that given by the first model, i.e. $z \neq 1$, $\forall x \in \Omega$.

On the other hand, even if the region near the surface where the transformation occurs is overestimated (with both models) in comparison with the experimental data obtained on the TSTs in the wheel/rail contact area where the maximum depth of TSTs hardly ever exceeded $10^{-1} \mathrm{~mm}$ in practice (see [19]) - the problem studied here does not take into account neither nonlinear contact with the dry and lubricated friction (see e.g. [43]) nor the strong 


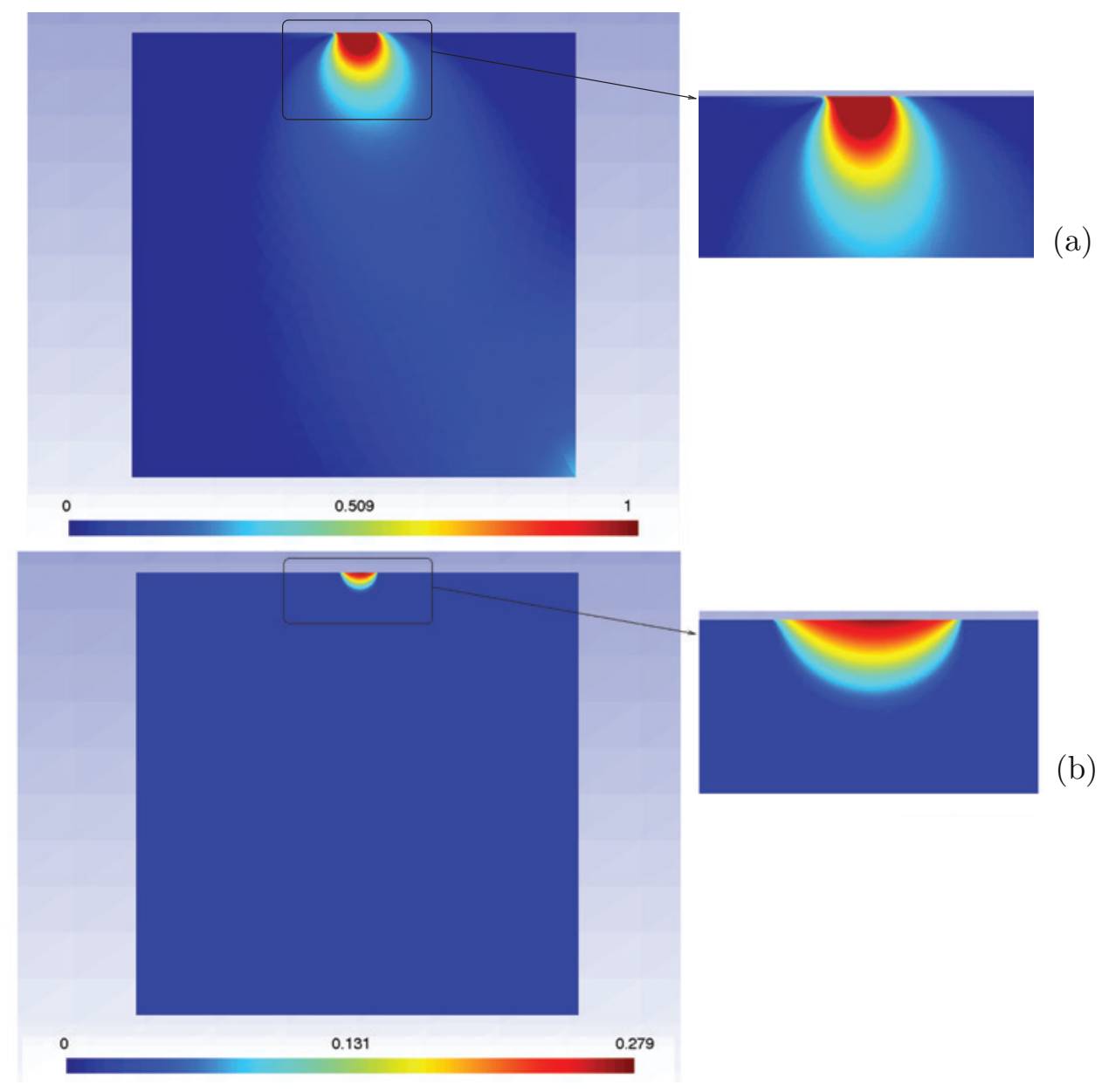

Figure 7: $z$-field for thermal-mechanical problem PB2 at time $t=10 \mathrm{~s}$ predicted by the first model (a) and the second model (b).

multiphysics coupling (see e.g. [44]) - here, thermomechanical type - which can certainly play a role in TST predictions.

The obtained results here confirm the numerical abilities of RMAs developed for use with the proposed thermomechanical models for predicting quasi-surface irreversible solid-solid phase transformations in the presence of two plasticity mechanisms. These different simulations are carried out using a modified Newton-Raphson procedure with a constant tangent operator (here, the elastic operator, see Section 3.2) in a total number of 16 incremental step with each model (where the number of incremental steps is chosen for providing a suitable convergence for each step and therefore do not perform a substep procedure). The Euclidean relative norm of the residual force vector $\frac{\left\|\left\{R_{n+1}^{(k+1)}\right\}\right\|}{\left\|\left\{R_{n+1}^{(k)}\right\}\right\|}$ with a tolerance $p_{T O L}=10^{-6}$ resulted in the convergence of the finite-element solution i.e.: $\frac{\left\|\left\{R_{n+1}^{(k+1)}\right\}\right\|}{\left\|\left\{R_{n+1}^{(k)}\right\}\right\|} \leq 10^{-6}$ (see [45]). The convergence of the relative norm associated with the residual vector at five incremental steps out of 16 performed with each model is shown in Tables 1 and 2 (with the first and second models, respectively). The results presented in Table 1 clearly show the occurrence of a non-quadratic convergence process (with the elastic operator) for residual norm in the case of the first model. Those presented in Table 2 show that although the elastic operator is used in the second model, it gives a satisfactory convergence rate. Indeed, the non-linear processes involved when the first model is used are higher than in the case of the second model which means that the use of the elastic operator with the second model has sufficed to obtain a suitable convergence rate (i.e. quasi-quadratic), which is not the case with the first model. Based on both these non-linear processes and the use of an elastic operator, the first model required a reasonable maximum number of iterations during all the incremental steps in order to converge towards the solution of the displacement vector associated with the problem studied. 


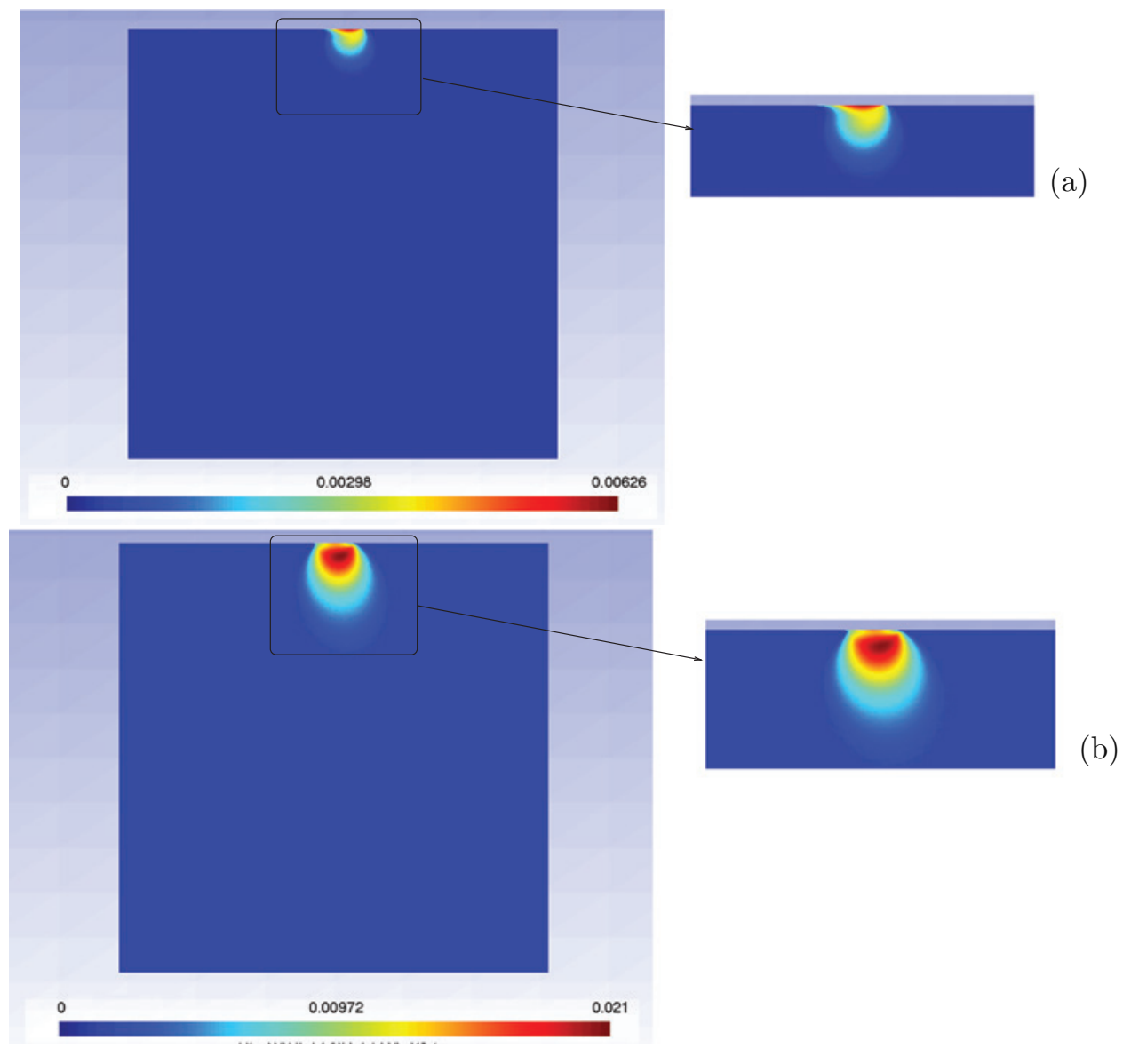

Figure 8: $v$-field for thermal-mechanical problem PB2 at time $t=10 \mathrm{~s}$ predicted by the first model (a) and the second model (b).

Table 1: Relative norm of the residual force vector $\frac{\left\|\left\{R_{n+1}^{(k+1)}\right\}\right\|}{\left\|\left\{R_{n+1}^{(k)}\right\}\right\|}$ at some of the steps performed with the first model.

\begin{tabular}{lrrrrr}
\hline Iteration (k) & Third step & Fifth step & Seventh step & Eleventh step & Fourteen step \\
\hline 0 & $6.4901 \mathrm{E}-02$ & $1.2631 \mathrm{E}-01$ & $2.9511 \mathrm{E}-02$ & $7.0043 \mathrm{E}-02$ & $1.2664 \mathrm{E}-01$ \\
1 & $3.7643 \mathrm{E}-03$ & $9.3909 \mathrm{E}-03$ & $6.7415 \mathrm{E}-04$ & $4.1950 \mathrm{E}-03$ & $1.3985 \mathrm{E}-02$ \\
2 & $3.2192 \mathrm{E}-04$ & $4.7753 \mathrm{E}-03$ & $5.8018 \mathrm{E}-05$ & $1.5301 \mathrm{E}-03$ & $9.4971 \mathrm{E}-03$ \\
3 & $3.8773 \mathrm{E}-05$ & $4.4985 \mathrm{E}-03$ & $4.8591 \mathrm{E}-05$ & $7.3359 \mathrm{E}-04$ & $7.8342 \mathrm{E}-03$ \\
4 & $6.2836 \mathrm{E}-05$ & $3.6453 \mathrm{E}-03$ & $1.2140 \mathrm{E}-06$ & $1.8236 \mathrm{E}-04$ & $7.0522 \mathrm{E}-03$ \\
5 & $1.0178 \mathrm{E}-06$ & $3.6556 \mathrm{E}-03$ & $3.5130 \mathrm{E}-08$ & $1.3221 \mathrm{E}-05$ & $8.6781 \mathrm{E}-04$ \\
6 & $1.6449 \mathrm{E}-07$ & $2.5014 \mathrm{E}-03$ & & $1.1421 \mathrm{E}-06$ & $1.2036 \mathrm{E}-04$ \\
7 & & $3.1181 \mathrm{E}-04$ & & $1.0488 \mathrm{E}-07$ & $1.7778 \mathrm{E}-05$ \\
8 & & $4.2088 \mathrm{E}-05$ & & & $2.6261 \mathrm{E}-06$ \\
9 & & $5.8927 \mathrm{E}-06$ & & & $3.9029 \mathrm{E}-07$ \\
10 & & $8.4919 \mathrm{E}-07$ & & & \\
\hline
\end{tabular}

\section{Conclusion}

In this paper, a numerical procedure is presented, based on RMA for dealing with two-yield surface thermoviscoplastic constitutive models. The two thermomechanical models developed in the consistent continuum framework can be used for predicting the quasi-surface irreversible solid-solid phase transformations which can occur in some practical problems such as TSTs on the railroad network's rails, for example. Apart from the classical plastic behaviour which always occurs in metallic materials, these models account for TRIP-like processes occurring 
Table 2: Relative norm of the residual force vector $\frac{\left\|\left\{R_{n+1}^{(k+1)}\right\}\right\|}{\left\|\left\{R_{n+1}^{(k)}\right\}\right\|}$ at some of the steps performed with the second model.

\begin{tabular}{lrrrrr}
\hline Iteration (k) & Third step & Fifth step & Seventh step & Eleventh step & Fourteenth step \\
\hline 0 & $8.2293 \mathrm{E}-04$ & $8.0649 \mathrm{E}-04$ & $3.0661 \mathrm{E}-03$ & $2.6114 \mathrm{E}-03$ & $5.9755 \mathrm{E}-04$ \\
1 & $1.9353 \mathrm{E}-06$ & $2.8524 \mathrm{E}-06$ & $3.6353 \mathrm{E}-05$ & $3.5783 \mathrm{E}-05$ & $4.5425 \mathrm{E}-07$ \\
2 & $5.5642 \mathrm{E}-16$ & $5.5643 \mathrm{E}-16$ & $3.7097 \mathrm{E}-16$ & $5.5651 \mathrm{E}-16$ & \\
\hline
\end{tabular}

during solid-solid phase transformations under mechanical loading conditions. In order to simulate these two processes numerically, a complete algorithmic treatment is presented, based on the use of two rate-dependent constitutive models along with the associated CTO. The numerical abilities associated with the algorithmic procedures used in the proposed models are tested, assessed and discussed on some examples. The obtained results show that these models can be able to predict the quasi-surface irreversible solid-solid phase transformations involving two plasticity type processes.

\section{A Comments}

\section{A.1 For Section 2}

(S2)-(i) Concerning the yield functions associated with TRIP-like processes, we consider a DruckerPrager type (see [33]) for the first model and a non-standard type for the second model (which is based on the observed physical arguments by [18]).

(S2)-(ii) For the internal state variables associated with classical viscoplastic $\left(\boldsymbol{e}^{p c}\right)$ and TRIP-like $\left(\boldsymbol{e}^{p z}\right.$ and $z$ ) processes, an "associative" and a "nonassociative" flow rule are adopted (eqs. (4)- (5)) (see [33, 34]).

(S2)-(iii) The two thermomechanical models presented here are thermodynamically consistent (see [17, 32]), i.e. they comply with the second principle of thermodynamics (see [34]).

\section{A.2 For Section 3}

(S3)-(i) A strong thermomechanical coupling exists between the thermal and mechanical evolutions through $\dot{T}$ and $\dot{\sigma}$ and are not taken into account here in the numerical implementation. Therefore, the source terms present in the heat equation - corresponding to the thermoelastic, classical plastic and irreversible solid-solid phase transformation processes - are neglected. Indeed, throughout this section, we consider only a weak thermomechanical coupling, i.e. the thermal effects affect unilaterally the mechanical evolution and this leads to the temperature $T_{n}$ and its increment, $\Delta T_{n+1}=T_{n+1}-$ $T_{n}$, are assumed to be known at time $t_{n}$.

(S3)-(ii) For the sake of numerical simplicity, the latent heat due to the solid-solid phase transformation is not taken into consideration here, i.e. $\delta=0$.

(S3)-(iii) The RMA procedures, used with for these two models, are based on the following two-fold condition (see [32]):

$$
\begin{cases}f^{p z}\left(\boldsymbol{\sigma}_{n+1}^{e l,(k)}, p_{n} ; T_{n+1}\right)-f^{p z}\left(\boldsymbol{\sigma}_{n+1}^{(k)}, p_{n}\right. & \\ \left.+\Delta p_{n+1}^{(k)} ; T_{n+1}\right) \geq 0 & \left(1^{s t} \text { model }\right) \\ f^{p z}\left(T_{n+1}, P_{n+1}^{e l,(k)}\right)-f^{p z}\left(T_{n+1}, P_{n+1}^{(k)}\right) \geq 0 & \left(2^{s d} \text { model }\right)\end{cases}
$$

and

$f^{p c}\left(\boldsymbol{\sigma}_{n+1}^{e l(k)}, v_{n} ; T_{n+1}\right)-f^{p c}\left(\boldsymbol{\sigma}_{n+1}^{(k)}, v_{n}+\Delta v_{n+1}^{(k)} ; T_{n+1}\right) \geq 0$

Using eq. (3), we can see that $f^{p z}$ (in the first model) and $f^{p c}$ are two convex functions with respect to the couple of variables $(\boldsymbol{\sigma}, p)$ and $(\boldsymbol{\sigma}, v)$, i.e.:

- in the case of classical plasticity process:

$$
\begin{gathered}
\frac{\partial f^{p c}}{\partial \boldsymbol{\sigma}}\left(\boldsymbol{\sigma}_{n+1}^{(k)}\right):\left(\boldsymbol{\sigma}_{n+1}^{e l(k)}-\boldsymbol{\sigma}_{n+1}^{(k)}\right)-\frac{\partial f^{p c}}{\partial p}\left(v_{n}+\Delta v_{n+1}^{(k)}\right) \Delta v_{n+1}^{(k)} \\
=\underbrace{\Delta v_{n+1}^{(k)}}_{\geq 0} \underbrace{\left[\frac{9 \mu}{2} \frac{\boldsymbol{s}_{n+1}^{(k)}: \boldsymbol{s}_{n+1}^{(k)}}{\left(\sigma_{n+1}^{e q,(k)}\right)^{2}}+h\right]}_{>0} \geq 0
\end{gathered}
$$

- in the case of TRIP-like process (for the first model): 


$$
\begin{gathered}
\frac{\partial f^{p z}}{\partial \boldsymbol{\sigma}}\left(\boldsymbol{\sigma}_{n+1}^{(k)}\right):\left(\boldsymbol{\sigma}_{n+1}^{e l(k)}-\boldsymbol{\sigma}_{n+1}^{(k)}\right)-\frac{\partial f^{p z}}{\partial p}\left(p_{n}+\Delta p_{n+1}^{(k)}\right) \Delta p_{n+1}^{(k)} \\
=\underbrace{\Delta p_{n+1}^{(k)}}_{\geq 0} \underbrace{\left[\frac{9 \mu}{2} \frac{\boldsymbol{s}_{n+1}^{(k)}: \boldsymbol{s}_{n+1}^{(k)}}{\left(\sigma_{n+1}^{e q,(k)}\right)^{2}}+9 \kappa \chi^{2}+\zeta^{2} \beta\right]}_{>0} \geq 0
\end{gathered}
$$

Therefore, we can see that eq. (31)-(a) and (31)-(c) are automatically found to have with the above property (eqs. (32) and (33)).

- in the case of second model, eq. (31)-(b) is also satisfied since that:

$$
\begin{aligned}
& P_{n+1}^{e l(k)}-P_{n+1}^{(k)}=\kappa \frac{\Delta z_{n+1}^{(k)}}{\zeta} \geq 0 \text { with } \Delta z_{n}^{(k)} \geq 0 \\
& \kappa>0 \text { and } \zeta>0
\end{aligned}
$$

(S3)-(iv) When RMA step [(ii)-(2)] has been checked, then the response of the model is pressure-dependent elasto(visco)plastic (see [11, 12]) due only to the TRIP-like process (Figure 1(a)). Therefore, the "trial" elastic stress tensor, $\boldsymbol{\sigma}_{n+1}^{e l}$, is subject to an "oblique projection" onto the yield surface $f^{p z}\left(\boldsymbol{\sigma}_{n+1}^{(k)}, p_{n+1} ; T_{n+1}\right)$. However, when RMA step [(ii)-(4)] is satisfied, then the model is elastoviscoplastic due to only the classical, isochoric plastic process (Figure 1(b)): the "trial" elastic stress deviator tensor, $\boldsymbol{s}_{n+1}^{e l}$, is subject here to a "normal projection" onto the yield surface $f^{p c}\left(\boldsymbol{\sigma}_{n+1}^{(k)}, v_{n+1} ; T_{n+1}\right)$. It is worth noting that the RMA is associated with case (b) of Figure 1 known as "Radial Return Algorithm” (see for example [46, 47])

(S3)-(v) The rate-dependent case is used in the RMAs with both models as follows:

$$
\frac{\Delta p_{n}^{(k)}}{\Delta t_{n+1}}=\left\{\begin{array}{l}
\frac{\left\langle f^{p z}\left(\boldsymbol{\sigma}_{n+1}^{(k)}, p_{n+1}^{(k)} ; T_{n+1}\right)\right\rangle^{+}}{\eta \bar{\sigma}_{i}} H\left(1-\zeta p_{n+1}^{(k)}\right) \quad\left(1^{\text {st }} \text { model }\right) \\
\frac{\left\langle 1-\zeta p_{n+1}^{(k)}\right\rangle^{+}}{\eta}\left\langle f^{p z}\left(T_{n+1}, P_{n+1}^{(k)}\right)\right\rangle^{+} H\left(P_{n+1}^{(k)}\right)\left(2^{n d} \text { model }\right)
\end{array}\right.
$$

and

$$
\frac{\Delta v_{n}^{(k)}}{\Delta t_{n+1}}=\frac{\left\langle f^{p c}\left(\boldsymbol{\sigma}_{n+1}^{(k)}, v_{n+1}^{(k)}\right)\right\rangle^{+}}{\xi \sigma_{y p}}
$$

Rate-independent cases, where the viscous effects are negligible, can be treated like rate-dependent cases when the characteristic times of the viscous effects tend to zero, i.e. $\eta \longrightarrow 0$ (in the case of TRIP-like processes) and $\xi \longrightarrow 0$ (in that of classical plasticity); see [33, 34, 48]. Therefore, the rate-independent inelastic case can be considered as a limiting case of rate-dependent inelastic (socalled viscoinelastic) [48], e.g. the classical instantaneous plasticity is a borderline case of the viscoplasticity.

In the rate-independent case (e.g. in classical plasticity), the elastic/plastic regime under loading/unloading condition is determined by Kuhn-Tucker relations such as:

$$
f \leq 0, \dot{\lambda} \geq 0 \text { and } \dot{\lambda} f=0
$$

In line with eq. (36), we can see that (i) an elastic regime is obtained when $f<0$ and $\dot{\lambda}=0$; (ii) an elastoplastic regime is achieved when $f=0$ and $\dot{\lambda} \geq 0$; (iii) a fully plastic regime is reached when $f=0$ and $\dot{\lambda}>0$. In the case of rate-independent plasticity (known as classical instantaneous plasticity), the plastic multiplier $\dot{\lambda}$ is obtained with the consistency condition: $f=\dot{f}=0$.

In order to use the same approach in the ratedependent case, the yield functions associated with TRIPlike and classical plasticity processes (eq. (35)) can be written in the following form (see [48]):

- $f^{p z}\left(\boldsymbol{\sigma}_{n+1}^{(k)}, p_{n+1} ; T_{n+1}\right) \leq 0$ in the first model (resp. $f^{p z}\left(T_{n+1}, P_{n+1}^{(k)}\right)=0$ in the second model) is replaced

$$
\begin{aligned}
& \text { by } \tilde{f}^{p z}\left(\boldsymbol{\sigma}_{n+1}^{(k)}, p_{n+1} ; T_{n+1}\right)=f^{p z}-\eta \bar{\sigma}_{i} \frac{\Delta p_{n+1}^{(k)}}{\Delta t_{n+1}} \leq 0 \text { (resp. } \\
& \left.\tilde{f}^{p z}\left(T_{n+1}, P_{n+1}^{(k)}\right)=f^{p z}-\frac{\eta}{\left(1-\zeta p_{n+1}^{(k)}\right)} \frac{\Delta p_{n+1}^{(k)}}{\Delta t_{n+1}} \leq 0\right) . \\
& -f^{p c}\left(\boldsymbol{\sigma}_{n+1}^{(k)}, v_{n+1}\right) \leq 0 \text { is replaced by } \tilde{f}^{p c}\left(\boldsymbol{\sigma}_{n+1}^{(k)}, v_{n+1}\right)=f^{p c}- \\
& \xi \sigma_{y p} \frac{\Delta v_{n+1}^{(k)}}{\Delta t_{n+1}} \leq 0
\end{aligned}
$$

(S3)-(vi) In the second model, a semi-explicit approach is used to implement the RMA. Indeed, an implicit approach is considered with RMA procedure, except when solving the consistency condition (where the approach is explicit):

$$
\frac{\Delta p_{n+1}^{(k)}}{\Delta t_{n+1}}=\frac{\left\langle 1-\zeta p_{n}\right\rangle^{+}}{\eta}\left\langle f^{p z}\left(T_{n+1}, P_{n}^{(k)}\right)\right\rangle^{+} H\left(P_{n}^{(k)}\right)
$$

(S3)-(vii) Figure 2 shows a flowchart with main steps for the numerical integration of the constitutive equations involved in two models.

(S3)-(viii) In the first model, and in the case of a thermoelastoviscoplastic iteration (i.e. case [(ii)-(2,3,4)]; see Section 3.1), the fourth-order tensor $\mathcal{P}$ has symmetry properties. In the classical thermoviscoplastic iteration (i.e. case [(ii)-(3)]; see Section 3.1) corresponding only to 
classical plasticity processes, the fourth-order tensor $\mathcal{P}$ has the same symmetry properties than the elastic operator $\mathcal{E}$, i.e. $\mathcal{P}$ is a fourth-order symmetric operator (see [39]).

(S3)-(ix) Figure 4 shows the geometrical interpretation of local CTO $\mathcal{T}_{n+1}^{(k)}$ in line with eq. (20) (see [39]).

\section{References}

[1] Q. S. Nguyen, On the elastic-plastic initial-boundary value problem and its numerical integration, Int. J. Numer. Methods Eng. 11 (1977), 817-832.

[2] J. C. Simo, R. L. Taylor, Consistent tangent operators for rate-independent elastoplasticity, Comput. Meth. Appl. Mech. Eng. 48 (1985), 101-118.

[3] J. C. Simo, R. L. Taylor, A return mapping algorithm for plane stress elastoplasticity, Int. J. Numer. Methods Eng. 22 (1986), 649-670.

[4] J. C. Simo, T. R. J. Hughes, Computational Inelasticity, Springer Verlag, New York, 1997.

[5] M. Ortiz, E. P. Popov, Accuracy and stability of integration algorithms for elastoplastic constitutive relations, Int. J. Numer. Meth. Eng. 21 (1985), 1561-1576.

[6] M. Ortiz, J. C. Simo, An analysis of a new class of integration algorithms for elastoplastic constitutive relations, Int. J. Numer. Meth. Eng. 23 (1986), 353-366.

[7] M. Bonnet, S. Mukherjee, Implicit BEM formulations for usual and sensitivity problems in elasto-plasticity using the consistent tangent operator concept, Int. J. Solids Struct. 33 (1996), 1395-1416.

[8] H. Poon, S. Mukherjee, M. Bonnet, Numerical implementation of a CTO-base implicit approach for the BEM solution of usual and sensitivity problems in elasto-plasticity, Eng. Anal. Boundary Elem. 22 (1998), 257-269.

[9] C. A. Vidal, R. B. Haber, Design sensitivity analysis for rate-independent elastoplasticity, Comput. Meth. Appl. Mech. Eng. 107 (1993), 393-431.

[10] N. Aravas, On the numerical integration of a class of pressure-dependent plasticity models, Int. J. Numer. Meth. Eng. 24 (1987), 1395-1416.

[11] Z. L. Zhang, On the accuracies of numerical implementation algorithms for Gurson-based pressure-dependent elastoplastic constitutive models, Comput. Meth. Appl. Mech. Eng. 121 (1995), 15-28.

[12] Z. L. Zhang, Explicit consistent tangent moduli with a return mapping algorithm for pressure-dependent elastoplasticity models, Comput. Meth. Appl. Mech. Eng. 121 (1995), 29-44.

[13] J. Clausen, L. Damkilde, L. Andersen, An efficient return algorithm for non-associated plasticity with linear yield criteria in principal stress space, Comput. Struct. 85 (2007), 1795-1807.

[14] M. Kleiber, Computational coupled non-associative thermoplasticity, Comput. Meth. Appl. Mech. Eng. 90 (1991), 943-967.

[15] J. C. Ju, Consistent tangent moduli for a class viscoplasticity, J. Eng. Mech. 116 (1990), 1764-1779.
[16] A. Eleöd, F. Oucherif, J. Devecz, Y. Berthier, Conception of numerical and experimental tools for study of the Tribological Transformation of Surface (TTS), Proceedings of Lubrication at the Frontier: The Role of the Interface and Surface Layers in the Thin Film and Boundary Regime 673-682, 1999.

[17] G. Antoni, T. Désoyer, F. Lebon, A combined thermo-mechanical model for Tribological Surface Transformations, Mech. Mater. 49 (2012), 92-99.

[18] G. Baumann, H. J. Fecht, S. Liebelt, Formation of white-etching layers on rail treads, Wear 191 (1996), 133-140.

[19] W. Österle, H. Rooch, A. Pyzalla, L. Wang, Investigation of white etching layers on rails by optical microscopy, electron microscopy, X-ray and synchrotron X-ray diffraction, Mater. Sci. Eng.: A 303 (2001), 150-157.

[20] G. Antoni, T. Désoyer, F. Lebon, A thermo-mechanical modelling of the Tribological Transformations of Surface, C.R. Mec. 337 (2009), 653-658.

[21] A. Boudiaf, L. Taleb, M. E. A. Belouchrani, Effects of the austenite grain size on transformation plasticity in a 35 NCD 16 steel, Int. J. Microstruct. Mater. Prop. 5 (2010), 338-353.

[22] M. Dalgic, A. Irretier, H-W Zoch, G. Lowisch, Transformation plasticity at different phase transformation of a through hardening bearing steel, Int. J. Microstruct. Mater. Prop. 3 (2008), 49-64.

[23] G. W. Greenwood, R. H. Johnson, The deformation of metals under small stresses during phase transformations, Proc. R. Soc. London, Ser. A: Math. Phys. Sci. 293 (1965), 403-422.

[24] T. Inoue, Mechanism of transformation plasticity and the unified constitutive equation for transformation-thermo-mechanical plasticity with some applications', Int. J. Microstruct. Mater. Prop. 5 (2010), 319-327.

[25] R. Quey, F. Barbe, H. Hoang, L. Taleb, Effect of the random spatial distribution of nuclei on the transformation plasticity in diffusively transforming steel, Int. J. Microstruct. Mater. Prop. 5 (2010), 354-364.

[26] J. B. Leblond, J. Devaux, J. C. Devaux, Mathematical modelling of transformation plasticity in steels - Case of ideal-plastic phases. Int. J. Plast. 5 (1989), 551-571.

[27] J. B. Leblond, J. Devaux, J. C. Devaux, Mathematical modelling of transformation plasticity in steels - Coupling with strain hardening phenomena, Int. J. Plast. 5 (1989), 573-591.

[28] L. Taleb, F. Sidoroff, A micromechanical modeling of the Greenwood-Johnson mechanism in transformation induced plasticity, Int. J. Plast. 19 (2003), 1821-1842.

[29] J. C. Videau, G. Cailletaud, A. Pineau, Modélisation des effets mécaniques des transformations de phases pour le calcul de structures, J. de Physique IV, Colloque C3, supplément au Journal de Physique III 4 (1994), 227-232.

[30] J. C. Videau, G. Cailletaud, A. Pineau, Experimental study of the transformation induced plasticity in a Cr-Ni-Mo-Al-Ti steel, Journal de Physique IV, Colloque C1, supplément au Journal de Physique III, 6 (1996), 465-474.

[31] G. Antoni, T. Désoyer, F. Lebon, Tribological Transformations of Surface: a thermo-mechanical modelling, Proceedings The Tenth International Conference on Computational Structures Technology, CD-ROM, 14p, 2010.

[32] G. Antoni, Transformation Tribologique de Surface: une approche thermo-mécanique, $\mathrm{PhD}$ thesis, Université de Provence, France, 2010. 
[33] J. Besson, G. Cailletaud, J. L. Chaboche, S. Forest 'Mécanique non linéaire des matériaux', Hermès Science Publications, France, 2001.

[34] J. Lemaitre, J. L. Chaboche, Mechanics of Solid Materials, Cambridge University Press, United Kingdom, 1990.

[35] J. Garrigues, Fondements de la mécanique des milieux continus Hermes (2007).

[36] J. C. Simo, S. Govindjee, Exact closed-form solution of the return mapping algorithm in plane stress elasto-viscoplasticity, Eng. Comput. 5 (1988), 254-258

[37] J. C. Simo, S. Govindjee, B-Stability and symmetry presering return mapping algorithms in plasticity and viscoplasticity, Int. J. Numer. Methods Eng. 31 (1991), 151-176.

[38] J. C. Simo, C. Miehe, Associative coupled thermoplasticity at finite strains: Formulation, numerical analysis and implementation, Comput. Meth. Appl. Mech. Eng. 98 (1992), 41-104.

[39] M. Bonnet, A. Frangi, Analyse des solides déformables par la méthode des éléments finis, Editions de l'école Polytechnique Palaiseau, France, 2007.

[40] M. Abbas, 'Algorithme non linéaire quasi-statique', Code_Aster Technical Note, R50301, France, 2013.

[41] R. Halama, Z. Poruba, Tangent modulus in numerical integration of constitutive relations and its influence on convergence of N-R method, Appl. Comput. Mech. 3 (2009), 27-38.

[42] R. Blaheta, O. Jakl, J. Stary, Displacement decomposition and parallelisation of the PCG method for elasticity problems, Int. J. Comput. Sci. Eng. 1 (2/3/4) (2005), 183-191.

[43] I. Ficza, M. Vaverka;, M. Hartl, Numerical solution of contact pressure in lubricated non-smooth point contact using convolution algorithms, Int. J. Comput. Sci. Eng. 9 (5/6) (2014), 457- 464.

[44] H. Busing, J. Willkomm, C. H. Bischof, C. Clauser, Using exact Jacobians in an implicit Newton method for solving multiphase flow in porous media, Int. J. Comput. Sci. Eng. 9 (5/6) (2014), 499-508.

[45] M. R. Gosz, Finite Element Method: Applications in Solids, Structures, and Heat Transfer, CRC Press, 2005.

[46] G. H. Paulino, Y. Liu, Implicit consistent and continuum tangent operators in elastoplastic boundary element formulations, Comput. Meth. Appl. Mech. Eng. 190 (2001), 2157-2179.

[47] G. Widlak, Radial Return method applied in thick-walled cylinder analysis, J. Theor. Appl Mech. 48 (2010), 381-395.

[48] Nukala, P. K. V. V., A return mapping algorithm for cyclic viscoplastic constitutive models. Comput Meth. Appl. Mech. Eng. 195 (2006), 148-178. 